\title{
CATEgorification of a ReCURsive Formula for Kazhdan-Lusztig POLYNOMials
}

\section{DAVID PlazA}

\author{
Universidad de Chile, \\ Facultad de Ciencias, \\ Santiago, Chile
}

Email: davidricardoplaza@gmail.com

\begin{abstract}
We obtain explicit branching rules for graded cell modules and graded simple modules over the endomorphism algebra of a Bott-Samelson bimodule. These rules allow us to categorify a well-known recursive formula for Kazhdan-Lusztig polynomials.
\end{abstract}

\section{INTRODUCTION}

Let $(W, S)$ be a Coxeter system. Let $l: W \rightarrow \mathbb{Z}_{\geq 0}$ be the corresponding length function. We denote by $\leq$ to the usual Bruhat order on $W$. Kazhdan and Lusztig (KL) introduced a family of polynomials with integer coefficients that are indexed using pairs of elements of $W$ KL79. These polynomials are now known as KL polynomials and can be defined in multiple equivalent ways. The definition provided here best suits our purposes and involves a recursive process. For $x, w \in W$, we define the KL polynomial, denoted $P_{x, w} \in \mathbb{Z}[q]$, as follows. First, we set

$$
P_{x, w}=0 \text { if } x \not \leq w \text {, and } P_{x, w}=1 \text { if } x=w .
$$

Let $e$ be the identity element of $\mathrm{W}$. Recall that $e \leq x$, for all $x \in W$. Therefore, $P_{x, e}$ is determined by (1.1), for all $x \in W$. Now, fix $w \in W$ and assume that $P_{u, v}$ has been defined, for all $u \in W$ and $v<w$. For $x<w$ and $s \in S$, satisfying $l(s w)<l(w)$, we define

$$
c=c_{s}(x):= \begin{cases}0, & \text { if } x<s x \\ 1, & \text { if } x>s x\end{cases}
$$

Then, $P_{x, w}$ is defined as

$$
P_{x, w}=q^{c-1} P_{s x, s w}+q^{c} P_{x, s w}-\sum_{s z<z<s w} \mu(z, s w) q^{(l(z)-l(w)) / 2} P_{x, z}
$$

where $\mu(z, s w)$ is the coefficient of $q^{(l(s w)-l(z)-1) / 2}$ in $P_{z, s w}$. It was proven in HU92 that this definition is equivalent to the original definition given by Kazhdan and Lusztig in [KL79].

The main result in this paper is a categorification of (1.3). The rest of this section provides a precise explication about what is meant by a categorification of (1.3). Roughly speaking, we refer to a categorification of (1.3) as the process that gives (1.3) using the category of Soergel bimodules.

For every Coxeter system $(W, S)$, Soergel $[\mathbf{S 9 2}$ constructed a category, $\mathcal{B}=\mathcal{B}(W, S, V, \mathbb{R})$, of $\mathbb{Z}$-graded bimodules over a polynomial ring $R$ with coefficients in the real numbers $\mathbb{R}$. It depends on $(W, S)$ and on a finite-dimensional 
$\mathbb{R}$-representation $V$ of $W$. Soergel proved that (up to a degree shift) $W$ parameterizes the set of indecomposable objects in $\mathcal{B}$. We let $B_{w} \in \mathcal{B}$ denote the corresponding indecomposable object. Soergel [S07] also proved that $\mathcal{B}$ is a categorification of the Hecke algebra $\mathcal{H}$ of $W$. This means that there exists an algebra isomorphism $\eta:[\mathcal{B}] \rightarrow \mathcal{H}$ between the split Grothendieck group $[\mathcal{B}]$ of $\mathcal{B}$ and $\mathcal{H}$. Soergel proposed the following conjecture, which came to be known as Soergel's conjecture:

$$
\eta\left(\left[B_{w}\right]\right)=\underline{H}_{w},
$$

where $\left\{\underline{H}_{w}\right\}_{w \in W}$ is the KL basis of $\mathcal{H}$. In 2014, Elias and Williamson EW14 proved this conjecture. We should mention that, by applying Soergel's previous work, their results provide a proof for the longstanding KL positivity conjecture for every Coxeter system. In a previous publication [P14, the author proved that Soergel's conjecture also implies a related conjecture for KL polynomials, known as the monotonicity conjecture.

The category of Soergel bimodules can be obtained as the graded Karoubi envelope of another category, BBSBim, of $\mathbb{Z}$-graded $(R, R)$-bimodules, known as Bott-Samelson bimodules. For each expression $\underline{w}=\left(s_{1}, s_{2}, \ldots, s_{k}\right) \in S^{k}$, we have a Bott-Samelson bimodule $B S(\underline{w})$ (for details see Section 2.2). Let $R^{+}$be the ideal of $R$ generated by homogeneous elements of nonzero degree. As $\mathbb{R} \cong R / R^{+}$we can view $\mathbb{R}$ as a left $R$-module. The endomorphism ring $\operatorname{End}(B S(\underline{w}))$ of a Bott-Samelson bimodule $B S(\underline{w})$ can be naturally equipped with a $(R, R)$-bimodule structure. Therefore, we can define the $\mathbb{R}$-algebra $A_{\underline{w}}:=\operatorname{End}(B S(\underline{w})) \otimes_{R} \mathbb{R}$. The algebra $A_{\underline{w}}$ is a graded cellular $\mathbb{R}$-algebra in the sense of $\mathrm{Hu}$ and Mathas [HM10, with a graded cellular basis, the double leaves basis. This basis was defined by Libedinsky in [L15] and it generalizes his previous construction of the light leaves basis [L08].

The existence of a graded cellular basis for $A_{\underline{w}}$ allows us to define graded (right) cell modules and graded (right) simple modules, as well as graded decomposition numbers. If $\underline{w}$ is a reduced expression of an element $w \in W$, then the set of graded cell modules for $A_{\underline{w}}$ is parameterized by

$$
\Lambda(\underline{w}):=\{x \in W \mid x \leq w\} .
$$

Given $x \in \Lambda(\underline{w})$, we use $\Delta_{\underline{w}}(x)$ to denote the corresponding graded cell module. Graded simple modules are parameterized by a subset $\Lambda_{0}(\underline{w})$ of $\Lambda(\underline{w})$. For $y \in \Lambda_{0}(\underline{w})$, we denote by $D_{\underline{w}}(y)$ the corresponding graded simple module. The set $\left\{D_{\underline{w}}(y) \mid y \in \Lambda_{0}(\underline{w})\right\}$ is a complete set (up to a degree shift) of pairwise non-isomorphic graded simple right $A_{\underline{w}}$-modules. We also let $d_{\underline{w}}(x, y) \in \mathbb{Z}\left[v, v^{-1}\right]$ denote the corresponding graded decomposition number. We recall that $\bar{d}_{\underline{w}}(x, y)$ counts the number of times $D_{\underline{w}}(y)$ appears in a graded composition series of $\Delta_{\underline{w}}(x)$. Because Soergel's conjecture proved to be valid, these graded decomposition numbers coincide with the KL polynomials when the former are defined and the latter are suitably normalized (see Proposition 4.8).

For the rest of this section we fix an element $w \in W$ and a reduced expression $\underline{w}=\left(s_{1}, s_{2}, \ldots, s_{k}\right)$ of $w$. The expression $\underline{w^{\prime}}=\left(s_{2}, \ldots, s_{k}\right)$ is a reduced expression for $w^{\prime}:=s_{1} w \in W$. The algebra $A_{\underline{w^{\prime}}}$ can be naturally embedded into $A_{\underline{w}}$. Accordingly, each $A_{\underline{w}}$-module can be considered as a $A_{\underline{w^{\prime}}}$-module by restriction. Given a graded $A_{\underline{\underline{w}}}$ module $M$, we let $\operatorname{Res}(M)$ represent its restriction. In particular, we can restrict graded cell modules and graded simple modules of $A_{\underline{w}}$. In this paper, we obtain explicit graded branching rules for these modules. In other words, we show how the graded cell modules and the graded simple modules of $A_{\underline{w}}$ decompose (when they are viewed as $A_{\underline{w^{\prime}}}$-modules) in terms of graded cell modules and graded simple modules of $A_{\underline{w^{\prime}}}$, respectively.

A concise way to express these branching rules is in terms of graded Grothendieck groups. Let $A$ be a finite dimensional $\mathbb{Z}$-graded algebra. The graded Grothendieck group $\mathcal{G}(A)$ of $A$ is the $\mathbb{Z}\left[v, v^{-1}\right]$-module generated by symbols $[M]$, where $M$ runs over the finite dimensional graded right $A$-modules, with relations:

1. $[M\langle k\rangle]=v^{k}[M]$, for all graded right $A$-module $M$ and $k \in \mathbb{Z}$;

2. $[M]=\left[M^{\prime}\right]+\left[M^{\prime \prime}\right]$ if there exists a short exact sequence $0 \rightarrow M^{\prime} \rightarrow M \rightarrow M^{\prime \prime} \rightarrow 0$ of graded right $A$-modules. 
In particular, for $A=A_{\underline{w}}\left(\right.$ resp. $\left.A=A_{\underline{w^{\prime}}}\right)$, we write $\mathcal{G}_{\underline{w}}:=\mathcal{G}\left(A_{\underline{w}}\right)\left(\right.$ resp. $\left.\mathcal{G}_{\underline{w^{\prime}}}:=\mathcal{G}\left(A_{\underline{w^{\prime}}}\right)\right)$. Using this terminology, the graded branching rules can be expressed as follows:

Theorem A. There exists a homomorphism of $\mathbb{Z}\left[v, v^{-1}\right]$-modules Res $: \mathcal{G}_{\underline{w}} \rightarrow \mathcal{G}_{\underline{w^{\prime}}}$ determined by

$$
\operatorname{Res}([M])=[\operatorname{Res}(M)],
$$

for every right graded $A_{\underline{w}}$ module $M$. The image of the class of the cell module $\Delta_{\underline{w}}(x)$ under this homomorphism is

$$
\operatorname{Res}\left(\left[\Delta_{\underline{w}}(x)\right]\right)=\left\{\begin{aligned}
v^{-1}\left[\Delta_{\underline{w}^{\prime}}(x)\right]+\left[\Delta_{\underline{w^{\prime}}}\left(s_{1} x\right)\right], & \text { if } s_{1} x<x \\
v\left[\Delta_{\underline{w^{\prime}}}(x)\right]+\left[\Delta_{\underline{w^{\prime}}}\left(s_{1} x\right)\right], & \text { if } s_{1} x>x .
\end{aligned}\right.
$$

On the other hand, if $y \in \Lambda_{0}(\underline{w})$, then the restriction of the simple module $D_{\underline{w}}(y)$ is given by

$$
\operatorname{Res}\left(\left[D_{\underline{w}}(y)\right]\right)=\sum_{u \in \Lambda_{0}\left(\underline{w^{\prime}}\right)} h_{s_{1}, u}^{y}\left[D_{\underline{w^{\prime}}}(u)\right],
$$

where $h_{s_{1}, u}^{y} \in \mathbb{Z}\left[v, v^{-1}\right]$ denotes the coefficient of $\underline{H}_{y}$ in the expansion of $\underline{H}_{s_{1}} \underline{H}_{u}$ in terms of the $K L$ basis of $\mathcal{H}$.

From the definition of Grothendieck group, we know that $\mathcal{C}_{\underline{w}}:=\left\{\left[D_{\underline{w}}(y)\right] \mid y \in \Lambda_{0}(\underline{w})\right\}$ is a $\mathbb{Z}\left[v, v^{-1}\right]$-basis of $\mathcal{G}_{\underline{w}}$. Furthermore, by definition of graded decomposition numbers, for $x \leq w$ the expansion of the class $\left[\Delta_{\underline{w}}(x)\right]$ in terms of the basis $\mathcal{C}_{\underline{w}}$ is given by

$$
\left[\Delta_{\underline{w}}(x)\right]=\sum_{y \in \Lambda_{0}(\underline{w})} d_{\underline{w}}(x, y)\left[D_{\underline{w}}(y)\right] .
$$

We can now apply the homomorphism Res to the equation (1.9). Theorem A provides two different ways to calculate this value. This allows us to obtain the following equation in $\mathcal{G}_{w^{\prime}}$.

$$
\sum_{u \in \Lambda_{0}\left(\underline{w^{\prime}}\right)}\left(\sum_{y \in \Lambda_{0}(\underline{w})} d_{\underline{w}}(x, y) h_{s_{1}, u}^{y}\right)\left[D_{\underline{w^{\prime}}}(u)\right]=\left\{\begin{aligned}
v^{-1}\left[\Delta_{\underline{w}^{\prime}}(x)\right]+\left[\Delta_{w^{\prime}}\left(s_{1} x\right)\right], & \text { if } s_{1} x<x \\
v\left[\Delta_{\underline{w^{\prime}}}(x)\right]+\left[\Delta_{\underline{w^{\prime}}}\left(s_{1} x\right)\right], & \text { if } s_{1} x>x .
\end{aligned}\right.
$$

Equating coefficients of the basis elements $\left[D_{\underline{w^{\prime}}}(u)\right] \in \mathcal{C}_{\underline{w^{\prime}}}$ yields

$$
\sum_{y \in \Lambda_{0}(\underline{w})} d_{\underline{w}}(x, y) h_{s_{1}, u}^{y}=\left\{\begin{aligned}
v^{-1} d_{\underline{w^{\prime}}}(x, u)+d_{\underline{w^{\prime}}}\left(s_{1} x, u\right), & \text { if } s_{1} x<x ; \\
v d_{\underline{w^{\prime}}}(x, u)+d_{\underline{w^{\prime}}}\left(s_{1} x, u\right), & \text { if } s_{1} x>x
\end{aligned}\right.
$$

for all $u \in \Lambda_{0}\left(\underline{w^{\prime}}\right)$. As we already mentioned, the graded decomposition numbers coincide with (a normalized version of) the KL-polynomials. Accordingly, equation (1.11) provides a relation between KL-polynomials. In general, determining which elements belong to $\Lambda_{0}\left(\underline{w^{\prime}}\right)$ is a hard task. However, we can straightforwardly note that $w^{\prime} \in \Lambda_{0}\left(\underline{w^{\prime}}\right)$. Equation (1.11) corresponding to the element $w^{\prime}$ is a normalized version of (1.3) (as we will see in Section 5). Summing up, we have produced (1.3) by studying the category of Soergel bimodules. We consider this procedure to be a categorification of (1.3).

The rest of this paper is organized as follows. In the next section, given an arbitrary Coxeter system, we introduce its corresponding Hecke algebra and Soergel bimodule category. We also show how to obtain a normalized version of (1.3). Section 3 reviews Libedinsky's construction of the double leaves basis. We recall that the double leaves basis is a graded cellular basis in Section 4 and then prove that the graded decomposition numbers coincide with the KL polynomials. Finally, in Section 5, we obtain graded branching rules for the graded cellular and graded simple modules. We conclude this section with a categorification of a normalized version of (1.3) using these branching rules. 
DAVID PLAZA

\section{Preliminaries}

In this section, given an arbitrary Coxeter group, we introduce its Hecke algebra and its corresponding category of Soergel bimodules. We discuss the relation of these subjects with the KL polynomials and also present a process for obtaining a normalized version of (1.3).

\subsection{Hecke algebras and Kazhdan-Lusztig polynomials}

Let $(W, S)$ be a Coxeter system with length function $l: W \rightarrow \mathbb{N}$, and let $e \in W$ denote the identity. We denote the order of $s t \in W$ by $m_{s t}=\{1,2, \ldots, \infty\}$, for all $s, t \in S$. The Hecke algebra $\mathcal{H}=\mathcal{H}(W, S)$ of $(W, S)$ is the $\mathbb{Z}\left[v, v^{-1}\right]$-algebra with generators $\left\{H_{s} \mid s \in S\right\}$ and relations

$$
\begin{gathered}
H_{s}^{2}=\left(v^{-1}-v\right) H_{s}+1 \text { for all } s \in S, \\
\underbrace{H_{s} H_{t} H_{s} \ldots}_{m_{s t} \text { times }}=\underbrace{H_{t} H_{s} H_{t} \ldots}_{m_{s t} \text { times }} \text { for all } s \neq t \in S .
\end{gathered}
$$

If $m_{s t}=\infty$, then the braid relation (2.2) is omitted. Throughout this paper, we use an underlined Roman letter to denote a finite sequence of elements in $S$, and we call such a sequence an expression. If $\underline{w}=s_{1} s_{2} \ldots s_{k}$ is an expression, then its length is $l(\underline{w})=k$. Omission of the underlining in an expression denotes the respective product in $W$. A expression $\underline{w}=s_{1} s_{2} \ldots s_{k}$ is reduced if $l(\underline{w})=l(w)$, where $l(w)$ is the length function of $W$. Differentiating between expressions and elements in $W$ is important because many of the concepts defined in this paper depend on expressions rather than elements in $W$.

Given an element $w \in W$ and a reduced expression $\underline{w}=s_{1} s_{2} \ldots s_{k}$ of $w$, we define $H_{w}=H_{s_{1}} H_{s_{2}} \ldots H_{s_{k}} \in \mathcal{H}$. It follows from (2.2) that $H_{w}$ is well defined, i.e., $H_{w}$ does not depend on the choice of a reduced expression for $w$. The set $\left\{H_{w} \mid w \in W\right\}$ is a basis of $\mathcal{H}$ as a $\mathbb{Z}\left[v, v^{-1}\right]$-module. We refer to this basis as the standard basis of $\mathcal{H}$. It follows from (2.1) that $H_{s}$ is invertible in $\mathcal{H}$, for all $s \in S$. Thus, $H_{w}$ is also invertible for every $w \in W$. Moreover, there exists a unique ring involution $d: \mathcal{H} \rightarrow \mathcal{H}$ determined by $d(v)=v^{-1}$ and $d\left(H_{w}\right)=H_{w^{-1}}^{-1}$, for all $w \in W$.

Theorem 2.1. (Kazhdan-Lusztig [KL79]) There exists a unique basis $\left\{\underline{H}_{w}\right\}_{w \in W}$ of $\mathcal{H}$ as a $\mathbb{Z}\left[v, v^{-1}\right]$-module that satisfies

$$
d\left(\underline{H}_{w}\right)=\underline{H}_{w}, \quad \text { and } \quad \underline{H}_{w}=H_{w}+\sum_{x<w} h_{x, w} H_{x},
$$

in which $h_{x, w} \in v \mathbb{Z}[v]$, and $<$ denotes the usual Bruhat order on $W$.

The set $\left\{\underline{H}_{w} \mid w \in W\right\}$ is called the Kazhdan-Lusztig basis of $\mathcal{H}$, and the polynomials $h_{x, w} \in \mathbb{Z}[v]$ are known as the Kazhdan-Lusztig polynomials.

Remark 2.2. We should note that this follows the normalization provided by Soergel in [S97] rather than the original normalization provided by Kazhdan and Lusztig in [KL79, which was used in the introduction. The two normalizations are related by $q=v^{-2}$, and the original Kazhdan-Lusztig polynomials $P_{x, w}(q) \in \mathbb{Z}[q]$ can be recovered from the Kazhdan-Lusztig polynomials $h_{x, w}(v) \in \mathbb{Z}[v]$ using

$$
h_{x, w}(v)=v^{l(w)-l(x)} P_{x, w}\left(v^{-2}\right) .
$$

Theorem 2.1provides an algorithm that can be used to calculate (inductively on the Bruhat order) the KL basis and the KL polynomials. The first KL basis elements can be easily defined as

$$
\underline{H}_{e}:=H_{e} \quad \text { and } \quad \underline{H}_{s}=: H_{s}+v, \quad \text { for all } s \in S .
$$


In order to continue this process, we must know the action of $\underline{H}_{s}$ on the standard basis of $\mathcal{H}$. This action is given by

$$
\underline{H}_{s} H_{x}= \begin{cases}H_{s x}+v H_{x}, & \text { if } s x>x ; \\ H_{s x}+v^{-1} H_{x}, & \text { if } s x<x,\end{cases}
$$

for all $x \in W$. Let us return to the calculation of the elements of the KL basis. Fix $u \in W$ and suppose $\underline{H}_{z}$ has been calculated, for all $z \leq u$. We choose $s \in S$ with $s u>u$ and write $\underline{H}_{s} \underline{H}_{u}$ in terms of the standard basis of $\mathcal{H}$ :

$$
\underline{H}_{s} \underline{H}_{u}=H_{s u}+\sum_{z<s u} g_{z} H_{z},
$$

for some $g_{z} \in \mathbb{Z}\left[v, v^{-1}\right]$. By applying (2.6) and from the fact that the KL polynomials have coefficients in $v \mathbb{Z}[v]$, we conclude that $g_{z} \in \mathbb{Z}[v]$. Hence, it is easy to see that

$$
\underline{H}_{s u}=\underline{H}_{s} \underline{H}_{u}-\sum_{z<s u} g_{z}(0) \underline{H}_{z}
$$

because the right side of (2.8) satisfies the conditions (2.3) provided in Theorem 2.1. Summing up, $\underline{H}_{s u}$ can be calculated using our previous knowledge of all the elements $\underline{H}_{z}$ of the KL basis in which $z \leq u$. We should note that this algorithm is far from efficient because a substantial amount of information is necessary for each new step in the algorithm.

On the other hand, equation (2.8) also provides an inductive procedure that can be used to compute the KL polynomials. Concretely, let $w=s u$ and choose $x \in W$ with $x<w$. Equating the coefficients of $H_{x}$ on both sides of (2.8) yields

$$
h_{x, w}=\left\{\begin{array}{rr}
v h_{x, s w}+h_{s x, s w}-\sum_{z<w} g_{z}(0) h_{x, z}, & \text { if } x<s x ; \\
v^{-1} h_{x, s w}+h_{s x, s w}-\sum_{z<w} g_{z}(0) h_{x, z}, & \text { if } x>s x .
\end{array}\right.
$$

In addition, (2.6) implies that $g_{z}(0) \neq 0$ only if $s z<z<s w$. Furthermore, equation (2.7), which defines the polynomials $g_{z}$, allows us to conclude that $g_{z}(0)$ equals the linear coefficient of the KL polynomial $h_{z, s w}$. We use $\mu(z, s w)$ to denote this value. Thus, we can rewrite (2.9) as

$$
h_{x, w}=\left\{\begin{array}{cl}
v h_{x, s w}+h_{s x, s w}-\sum_{s z<z<s w} \mu(z, s w) h_{x, z}, & \text { if } x<s x ; \\
v^{-1} h_{x, s w}+h_{s x, s w}-\sum_{s z<z<s w} \mu(z, s w) h_{x, z}, & \text { if } x>s x .
\end{array}\right.
$$

Note that we have already used the symbol $\mu(z, s w)$ in (1.3) to denote the coefficient of $q^{(l(s w)-l(z)-1) / 2}$ in the original KL polynomial $P_{z, s w}$. However, Remark 2.2 makes it clear that the linear coefficient of $h_{z, s w}$ coincides with the coefficient of $q^{(l(s w)-l(z)-1) / 2}$ in $P_{z, s w}$. This remark further indicates that (2.10) is a normalized version of (1.3). As mentioned in the introduction, the main result in this paper is a categorification of (1.3), or, equivalently, a categorification of (2.10). In actuality, we categorify (2.10) rather than (1.3) because the former is best suited for our purposes.

\subsection{The category of Soergel bimodules}

Recall that $(W, S)$ is an arbitrary Coxeter group. In order to introduce the category of Soergel bimodules, we must first fix a representation $V$ of $W$, which should satisfy certain technical requirements. 
DAVID PLAZA

Definition 2.3. A reflection faithful representation $V$ of $W$ over $\mathbb{R}$ is a finite-dimensional $\mathbb{R}$-representation of $W$ that satisfies the following conditions.

1. The representation $V$ is faithful.

2. If $V^{w}$ is the set of elements in $V$ fixed by $w \in W$, then $V^{w}$ has codimension 1 if and only if $w$ is conjugated to a simple reflection.

In [S07, Soergel demonstrated that such a representation exists for arbitrary Coxeter groups. Therefore, we can fix (for the rest of the paper) a reflection-faithful representation $V$ of $W$ defined over $\mathbb{R}$. Let $R$ be the $\mathbb{R}$-algebra of regular functions on $V$ with the following grading:

$$
R=\bigoplus_{i \in \mathbb{Z}_{\geq 0}} R_{i} \text {, with } R_{2}=V^{*} \text { and } R_{i}=0 \text { if } i \text { is odd. }
$$

We can think of the elements in $R$ as polynomials over $V^{*}$. We also define $R^{+}$to be the subring of $R$ generated by all homogeneous elements having a nonzero degree. It is easy to see that $\mathbb{R} \cong R / R^{+}$. This isomorphism will be frequently used in order to consider $\mathbb{R}$ as a left $R$-module.

Given a graded $(R, R)$-bimodule $B$ and $k \in \mathbb{Z}$, we let $B(k)$ signify the graded $(R, R)$-bimodule that is obtained from $B$ by shifting the grading by $k$. In other words, if $B=\bigoplus_{i \in \mathbb{Z}} B_{i}$ is a graded bimodule, then $B(k)_{i}=B_{k+i}$ for all $i \in \mathbb{Z}$. There is a natural action of $W$ on $R$, which is induced by the action of $W$ on $V$. For $s \in S$, let $R^{s}$ be the subring of $R$ fixed by $s$. The graded $(R, R)$-bimodule $B_{s}$ is defined to be

$$
B_{s}=R \otimes_{R^{s}} R(1),
$$

where the left (resp. right) action of $R$ on $B_{s}$ is given by left (resp. right) multiplication. Given any expression $\underline{w}=s_{1} s_{2} \ldots s_{k}$, we define the Bott-Samelson bimodule $B S(\underline{w})$ to be

$$
B S(\underline{w})=B_{s_{1}} \otimes_{R} B_{s_{2}} \otimes_{R} \ldots \otimes_{R} B_{s_{k}} .
$$

We have the following $(R, R)$-bimodule isomorphism.

$$
B S(\underline{w}) \cong R \otimes_{R^{s_{1}}} R \otimes_{R^{s_{2}}} \ldots \otimes_{R^{s_{k}}} R(k)
$$

Therefore, we can write any element of this module as a sum of terms given by $k+1$ polynomials in $R$, one in each slot, separated by the tensors. Denote by $\mathbb{B S B i m}$ the category whose objects include all direct sums and grading shifts of Bott-Samelson bimodules and whose morphisms are degree-preserving bimodule homomorphisms. For two objects $B, B^{\prime} \in \mathbb{B S B i m}$, let $\operatorname{Hom}\left(B, B^{\prime}\right)$ be the respective morphisms space. We also define

$$
\operatorname{Hom}^{\mathbb{Z}}\left(B, B^{\prime}\right)=\bigoplus_{k \in \mathbb{Z}} \operatorname{Hom}\left(B(k), B^{\prime}\right) .
$$

Finally, we define the category of Soergel bimodules, $\mathbb{S B i m}$, as the Karoubi envelope of $\mathbb{B S B i m}$. In other words, the objects in SBim are direct sums and graded shifts of direct summands of the objects in BSBim. In S07, Theorem 6.16], Soergel demonstrated that the indecomposable objects in SBim are indexed using elements of $W \times \mathbb{Z}$. They are written as $B_{w}(k)$ for $w \in W$ and $k \in \mathbb{Z}$. An indecomposable bimodule $B_{w}$ is completely determined by one property: it appears as a direct summand of $\mathbb{B S}(\underline{w})$ for every reduced expression $\underline{w}$ of $w$, and it does not appear as a direct summand of $\mathbb{B} \mathbb{S}(\underline{x})$ for any expression $\underline{x}$ with length less than $l(w)$.

Denote by $[\mathbb{S B i m}]$ the split Grothendieck group of $\mathbb{S B i m}$. That is, [SBim] is the abelian group generated by $[B]$ for every object $B \in \mathbb{S B i m}$, subject to the relation $[B]=\left[B_{1}\right]+\left[B_{2}\right]$ whenever $B \cong B_{1} \oplus B_{2}$. If we define $\left[B_{1}\right]\left[B_{2}\right]=\left[B_{1} \otimes B_{2}\right]$, then [SBim] is equipped with a ring structure. Furthermore, [SBim] has the structure of $\mathbb{Z}\left[v, v^{-1}\right]$-algebra by defining $v^{k}[B]=[B(k)]$, for all $k \in \mathbb{Z}$. The following result relates [SBim] with $\mathcal{H}$. It is known as Soergel's categorification theorem. 
Theorem 2.4. SOY, Theorem 1.10] There is a $\mathbb{Z}\left[v, v^{-1}\right]$-algebra isomorphism

$$
\epsilon: \mathcal{H} \rightarrow[\mathbb{S} B i m]
$$

that is uniquely determined by $\epsilon(v)=R(1)$ and $\epsilon\left(\underline{H}_{s}\right)=B_{s}$.

In order to describe an explicit inverse for $\epsilon$, we need to introduce standard bimodules. Given $x \in W$, we define the standard bimodule $R_{x}$ as the $(R, R)$-bimodule such that as left $R$-module $R_{x} \cong R$, and the right action of $R$ on $R_{x}$ is determined by deforming the usual right multiplication on $R$ by $x$, i.e.,

$$
r \cdot r^{\prime}:=r x\left(r^{\prime}\right) \text { for } r \in R_{x} \text { and } r^{\prime} \in R .
$$

Theorem 2.5. S07, Theorem 5.3] The homomorphism $\epsilon: \mathcal{H} \rightarrow[$ SBim] admits an inverse, $\eta:[\mathbb{S} B i m] \rightarrow \mathcal{H}$, given by

$$
\eta([B])=\sum_{x \in W} \operatorname{dim}_{v}\left(\operatorname{Hom}^{\mathbb{Z}}\left(B, R_{x}\right) \otimes_{R} \mathbb{R}\right) H_{x},
$$

where $\operatorname{dim}_{v}(-)$ signifies the respective graded dimension.

Theorem 2.5 implies that when we expand $\eta([B])$ in terms of the standard basis $\left\{H_{w}\right\}_{w \in W}$ of $\mathcal{H}$, the Laurent polynomials appearing in this expansion contain positive coefficients. The following result is known in literature as Soergel's Conjecture.

Theorem 2.6. EW14, Theorem 1.1] For any Coxeter system $(W, S)$, we have

$$
\epsilon\left(\underline{H}_{w}\right)=\left[B_{w}\right] .
$$

Consequently, $h_{x, y}=\operatorname{dim}_{v}\left(\operatorname{Hom}^{\mathbb{Z}}\left(B_{y}, R_{x}\right) \otimes_{R} \mathbb{R}\right)$, and the KL polynomials $h_{x, y}$ have positive coefficients.

\section{LIBEDINSKY DOUBLE LEAVES}

Let $\underline{w}$ and $\underline{v}$ be two expressions. In this section, we recall the construction of the double leaves basis (DLB) presented in [L15], which is a basis for $\operatorname{Hom}^{\mathbb{Z}}(B S(\underline{w}), B S(\underline{v}))$. An alternative description of the DLB, in terms of diagrams, can be found in EW13. It should be noted that, in our construction of the DLB, we work from right to left rather than applying the typical left-to-right approach.

Suppose $\alpha_{s} \in V^{*}$ is an equation that belongs to a hyperplane fixed by $s \in S$. Then, for all $s \in S$, we define the Demazure operator $\partial_{s}: R(2) \rightarrow R^{s}$ to be the morphism of graded $R^{s}$-modules given by

$$
\partial_{s}(f)=\frac{f-s \cdot f}{\alpha_{s}}
$$

In order to introduce the DLB, we first define a family of basic morphisms between Bott-Samelson bimodules. These morphisms are listed in Table 1, and their names, formulas, and degrees are provided. All elements belonging to the DLB can be constructed by compounding and tensoring the morphisms in this list. Therefore, the morphisms in Table 1 generate all the morphisms that exist between Bott-Samelson bimodules. This result is due to Libedinsky L08, Theorem 5.1].

In the last row of Table 1, $B_{s} B_{t} \ldots$ (resp. $B_{t} B_{s} \ldots$ ) represents the alternating tensor product of $B_{s}$ and $B_{t}$ (resp. $B_{t}$ and $B_{s}$ ) having $m_{s t}$ factors. Note that the formula column is empty for this row because an explicit formula (at least, not a simple formula) does not exist for this type of morphism. A morphism of type $f_{s t}$ can be uniquely determined as the only degree-zero morphism from $B_{s} B_{t} \ldots$ to $B_{t} B_{s} \ldots$, sending $1 \otimes 1 \ldots \otimes 1$ to $1 \otimes 1 \ldots \otimes 1$. 
DAVID PLAZA

\begin{tabular}{|l|l|c|}
\hline Name & Formula & Degree \\
\hline$m_{s}: B_{s} \rightarrow R$ & $1 \otimes 1 \rightarrow 1$ & 1 \\
\hline$e_{s}: R \rightarrow B_{s}$ & $1 \rightarrow \frac{1}{2}\left(\alpha_{s} \otimes 1+1 \otimes \alpha_{s}\right)$ & 1 \\
\hline$j_{s}: B_{s} \otimes_{R} B_{s} \rightarrow B_{s}$ & $1 \otimes f \otimes 1 \rightarrow \partial_{s}(f) \otimes 1$ & -1 \\
\hline$p_{s}: B_{s} \rightarrow B_{s} \otimes B_{s}$ & $1 \otimes 1 \rightarrow 1 \otimes 1 \otimes 1$ & -1 \\
\hline$f: R \rightarrow R$ & $1 \rightarrow f$ & $\operatorname{deg}(f)$ \\
\hline $\mathbb{I}_{s}: B_{s} \rightarrow B_{s}$ & $1 \otimes 1 \rightarrow 1 \otimes 1$ & 0 \\
\hline$f_{s t}: B_{s} B_{t} \ldots \rightarrow B_{t} B_{s} \ldots$ & & 0 \\
\hline
\end{tabular}

Table 1: Basic morphisms between Bott-Samelson bimodules

Remark 3.1. Subsequently, we exploit the symbol II in order to denote the identity of different Bott-Samelson bimodules. However, the subindex and superindex accompanying $\mathbb{I}$, as well as the context, should help avoid confusion.

For each expression $\underline{w}=s_{1} \ldots s_{n} \in S^{n}$, we inductively define a perfect binary directed tree, denoted as $\mathbb{T}_{\underline{w}}$, with nodes colored by Bott-Samelson bimodules and edges colored by morphisms from parent nodes to child nodes. In order to convert the construction of $\mathbb{T}_{\underline{w}}$ into an algorithm, we should establish the following.

Choices 3.2. $\quad$ 1. For each $x \in W$, we fix a reduced expression, which we write as $\underline{\mathbf{x}}$.

2. For each reduced expression $\underline{x}$ of $x \in W$, we fix a sequence of braid moves that converts $\underline{x}$ into $\underline{\mathrm{x}}$. Then, we denote by $F(\underline{x}, \underline{\mathbf{x}}): B S(\underline{x}) \rightarrow B S(\underline{\mathbf{x}})$ the morphism obtained by replacing each braid move in the above sequence by its corresponding morphism of type $f_{s r}$. In particular, we fix $F(\underline{\mathbf{x}}, \underline{\mathbf{x}})=\mathbb{I}_{\underline{\mathbf{x}}}$.

3. Let $x \in W$ and $s \in S$ with $l(s x)<l(x)$. Then, we fix a reduced expression $\underline{x}_{s}$ of $x$, which begins in $s$. We also set a sequence of braid moves that converts $\underline{\mathbf{x}}$ into $\underline{x}_{s}$. Finally, we fix a morphism $F\left(\underline{\mathbf{x}}, \underline{x}_{s}\right): B S(\underline{\mathbf{x}}) \rightarrow B S\left(\underline{x}_{s}\right)$ by replacing each braid move in the above sequence by its respective morphism of type $f_{s r}$.

Let us return to the construction of tree $\mathbb{T}_{\underline{w}}$. At depth 1, the tree is the one displayed in Figure 1

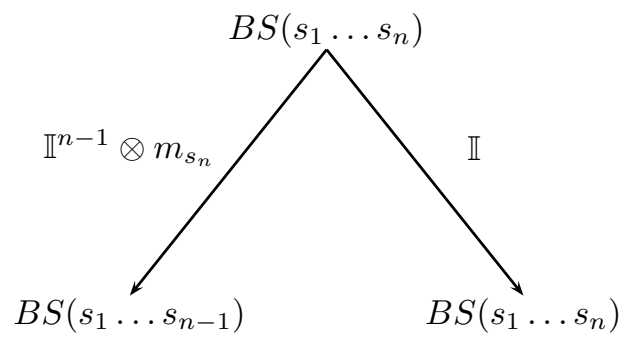

Figure 1: Level one of $\mathbb{T}_{\underline{w}}$

Suppose $1<k \leq n$, and assume we have constructed the tree to level $k-1$. Let $x \in W$ be such that a node $N$ of depth $k-1$ is colored by the Bott-Samelson bimodule $B S\left(s_{1} \ldots s_{n-k+1}\right) B S(\underline{\mathbf{x}})$. Then, two possibilities exist. 
a) $l\left(s_{n-k+1} x\right)>l(x)$. Figure 2 illustrates the construction of the child nodes and edges of $N$ for this case. Here, $s_{n-k+1} x$ represents the reduced expression of $s_{n-k+1} x$ obtained from $\underline{\mathbf{x}}$, in which $s_{n-k+1}$ has been positioned

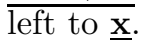

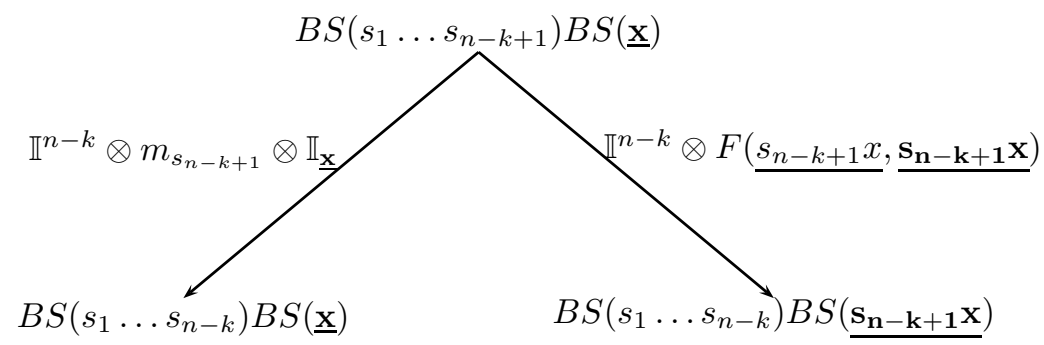

Figure 2: Level $k$ of $\mathbb{T}_{\underline{w}}$

b) $l\left(s_{n-k+1} x\right)<l(x)$. In this case, the child nodes of $N$ are colored by the two Bott-Samelson bimodules located at the bottom of Figure 3, and the child edges are colored by the morphisms obtained by composing the arrows. In this figure, the symbol $\underline{s}_{n-k+1} x$ represents the reduced expression of $s_{n-k+1} x$ obtained from $\underline{x}_{s_{n-k+1}}$ by dropping $s_{n-k+1}$ to the left of $\underline{x}_{s_{n-k+1}}$.

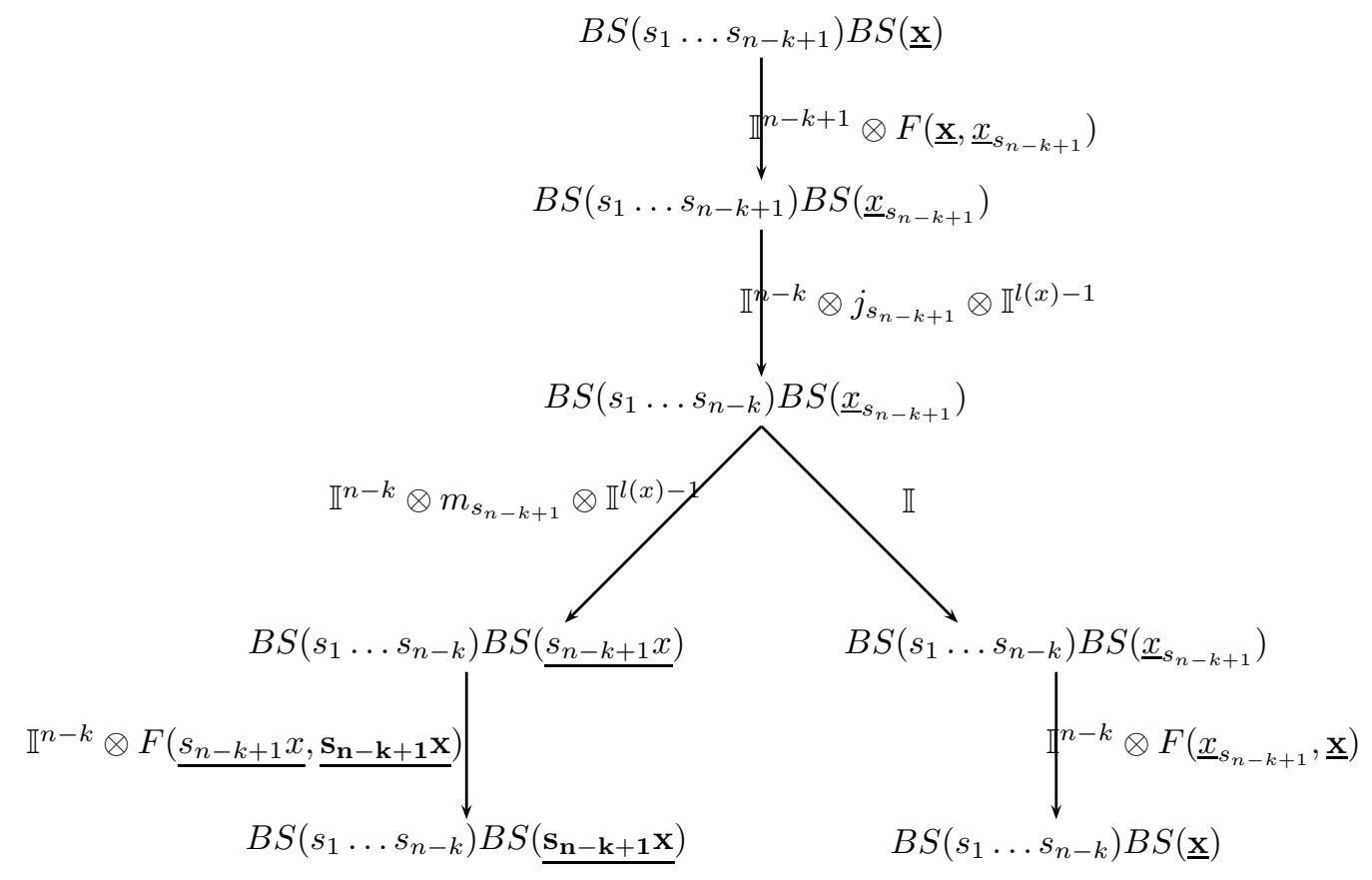

Figure 3: Level $k$ of $\mathbb{T}_{\underline{w}}$

This completes the construction of $\mathbb{T}_{\underline{w}}$. Examining this construction, we see that all leaves of the tree $\mathbb{T}_{\underline{w}}$ are colored by Bott-Samelson bimodules of form $B S(\underline{\mathbf{x}})$, where $\underline{\mathbf{x}}$ is the reduced expression fixed in Choices $3 . \overline{2}$ 
Furthermore, if $\underline{w}$ is a reduced expression of some element $w \in W$, the leaves in $\mathbb{T}_{\underline{w}}$ are colored by Bott-Samelson bimodules $B S(\underline{\mathbf{x}})$ with $x \leq w$.

Composing the corresponding arrows allows us to consider each leaf in $\mathbb{T}_{\underline{w}}$ that is colored by $B S(\underline{\mathbf{x}})$ as a morphism from $B S(\underline{w})$ to $B S(\underline{\mathbf{x}})$. Let $\mathbb{L}_{\underline{w}}(x)$ be the set of all leaves colored by $B S(\underline{\mathbf{x}})$. As mentioned earlier, we consider the set $\mathbb{L}_{\underline{w}}(x)$ to be a subset of $\operatorname{Hom}^{\mathbb{Z}}(B S(\underline{w}), B S(\underline{\mathbf{x}}))$. Note that every leaf is a homogeneous morphism because all leaves were constructed as compositions of homogeneous morphisms. In fact, the degree of each leaf can be computed using +1 or -1 for each occurrence of a morphism of type $m_{s}$ or $j_{s}$, respectively.

Remark 3.3. The set $\mathbb{L}_{\underline{w}}(x)$ is not uniquely determined because it relies heavily on Choices 3.2 . In other words, different choices produce different morphisms in $\mathbb{L}_{\underline{w}}(x)$. Thus, when we refer to this set, we must remember that we are considering a particular fixed choice for all the non-canonical steps in the construction of $\mathbb{T}_{\underline{w}}$. For each acceptable construction of $\mathbb{T}_{\underline{w}}$, the leaves of this tree can be used to build a double leaves basis. However, different choices produce different double leaves bases.

In order to complete the construction of the double leaves basis, we need to define the adjoint tree $\mathbb{T}_{\underline{w}}^{a}$ of $\mathbb{T}_{\underline{w}}$. First, we note that only morphisms of type $m_{s}, j_{s}$, and $f_{s r}$ were used in order to construct $\mathbb{T}_{\underline{w}}$. Given any leaf $l: B S(\underline{w}) \rightarrow B S(\underline{\mathbf{x}})$ in $\mathbb{T}_{\underline{w}}$, we define its adjoint leaf $l^{a}: B S(\underline{\mathbf{x}}) \rightarrow B S(\underline{w})$ by replacing each morphism $m_{s}, j_{s}$, and $f_{s r}$ in the construction of $l$ by $e_{s}, p_{s}$, and $f_{r s}$, respectively. Thus, we obtain an inverted tree, $\mathbb{T}_{\underline{w}}^{a}$, which has the same nodes as $\mathbb{T}_{\underline{w}}$ but arrows pointing in opposite directions.

Suppose $f \in \operatorname{Hom}^{\mathbb{Z}}(B S(\underline{w}), B S(\underline{u}))$ and $g \in \operatorname{Hom}^{\mathbb{Z}}(B S(\underline{x}), B S(\underline{y}))$. Then, we define

$$
f \cdot g= \begin{cases}f \circ g, & \text { if } \underline{w}=\underline{y} \\ \emptyset, & \text { if } \underline{w} \neq \underline{y}\end{cases}
$$

Given an expression $\underline{w}$, we use $\mathbb{L}_{\underline{w}}$ (resp. $\mathbb{L}_{\underline{w}}^{a}$ ) to indicate the set of all leaves in $\mathbb{T}_{\underline{w}}$ (resp. $\mathbb{T}_{\underline{w}}^{a}$ ). Finally, we are in a position to define the double leaves basis.

Theorem 3.4. [L15, Theorem 3.2] For all expressions $\underline{w}$ and $\underline{v}$, the set $\mathbb{L}_{\underline{v}}^{a} \cdot \mathbb{L}_{\underline{w}}$ is a basis of $\operatorname{Hom}^{\mathbb{Z}}(B S(\underline{w}), B S(\underline{v}))$ as right $R$-module. We call this set the double leaves basis (DLB).

Aside from their usefulness in defining the DLB, the leaves also describe a basis for spaces of type $\operatorname{Hom}^{\mathbb{Z}}\left(B S(\underline{w}), R_{x}\right)$. Note that spaces of this form appear in Theorem 2.5. These spaces are relevant because they provide an alternative description for the cell modules defined in the next section. In order to explore this further, however, we must first introduce a new morphism.

For all $s \in S$, consider the $(R, R)$-bimodule morphism $\beta_{s}: B_{s} \rightarrow R_{s}$, determined by $\beta_{s}(p \otimes q)=p s(q)$, for all $p, q \in R$. On the other hand, we have $R_{x} R_{y} \cong R_{x y}$, for all $x, y \in W$. Therefore, we can also define a morphism, denoted by $\beta_{\underline{x}}: B S(\underline{x}) \rightarrow R_{x}$, for all $x \in W$. Furthermore, let us define the set

$$
\mathbb{L}_{\underline{w}}^{\beta}(x)=\left\{\beta_{\underline{x}} \circ l \mid l \in \mathbb{L}_{\underline{w}}(x)\right\} \subset \operatorname{Hom}^{\mathbb{Z}}\left(B S(\underline{w}), R_{x}\right) .
$$

In keeping with Libedinsky's work, we call $\mathbb{L}_{\underline{w}}^{\beta}(x)$ the standard leaves basis. The following proposition justifies this name choice.

Proposition 3.5. [L15, Proposition 6.1] If $\underline{w}$ is an expression and $x \in W$, then $\mathbb{L}_{\underline{w}}^{\beta}(x)$ is an R-basis of $\operatorname{Hom}^{\mathbb{Z}}\left(B S(\underline{w}), R_{x}\right)$ as a right $R$-module.

Corollary 3.6. Assume $\underline{w}$ is a reduced expression for $w$, and let $x \in W$. Then, $\operatorname{Hom}^{\mathbb{Z}}\left(B S(\underline{w}), R_{x}\right) \neq 0$ if and only if $x \leq w$.

Proof: This result is a direct consequence of Proposition 3.5 if we note that $\mathbb{L}_{\underline{w}}(x) \neq \emptyset$ if and only if $x \leq w$. 
GRADED CELLULARITY AND THE MONOTONICITY CONJECTURE

\section{Cellularity and KL polynomials}

Throughout this section, we fix the reduced expression $\underline{w}$. We recall that $\mathbb{R}$ can be considered as a left $R$-module via the isomorphism $\mathbb{R} \cong R / R^{+}$. Define $A_{\underline{w}}:=\operatorname{End}^{\mathbb{Z}}(B S(\underline{w})) \otimes_{R} \mathbb{R}$. The $\mathbb{R}$-algebra $A_{\underline{w}}$ has a graded cellular algebra structure - a concept defined by Hu and Mathas [HM10, who extended the work of Graham and Lehrer GL96] - and the DLB as its graded cellular basis. We will not delve into the details of graded cellular algebras because concepts involving $A_{\underline{w}}$ can be explicitly described. For a proof of the graded cellularity of $A_{\underline{w}}$ the reader is referred to [EW13, Proposition 6.22] and [P14, Theorem 4.1].

Graded cellular algebras are equipped with a family of modules, which are known as graded cell modules and have a bilinear form. The quotient of a graded cell module by the radical of its bilinear form is zero or simple, and all simples can be obtained in this way. In this section, we define graded cell modules and graded simple modules for $A_{\underline{w}}$ in terms of the DLB, as well as the graded decomposition numbers. We conclude this section by proving that the graded decomposition numbers and KL polynomials coincide, which is a generalization of [P14, Theorem 4.8].

\subsection{Graded cell modules and graded simple modules}

In order to provide a precise description for the graded cell modules and graded simple modules, we first need a couple of definitions.

Definition 4.1. Given two expressions $\underline{w}$ and $\underline{v}$, and assuming $x \in W$, we say that a double leaf $l_{1}^{a} \circ l_{2} \in \mathbb{L}_{\underline{v}}^{a} \cdot \mathbb{L}_{\underline{w}}$ factors through $x$ whenever $l_{1} \in \mathbb{L}_{\underline{v}}(x)$ and $l_{2} \in \mathbb{L}_{\underline{w}}(x)$.

Definition 4.2. Suppose $\underline{w}$ and $\underline{v}$ are expressions. For $x \in W$, we define $\mathbb{D L}_{<x}(\underline{w}, \underline{v})$ to be the $R$-submodule of $\operatorname{Hom}^{\mathbb{Z}}(B S(\underline{w}), B S(\underline{v}))$ generated by double leaves in $\mathbb{L}_{\underline{v}}^{a} \cdot \mathbb{L}_{\underline{w}}$ that factor through $u<x$.

We are now in a position to define the graded cell modules for $A_{\underline{w}}$. Suppose $x \leq w, l \in \mathbb{L}_{\underline{w}}(x)$, and $a \in$ $\operatorname{End}^{\mathbb{Z}}(B S(\underline{w}))$. Then, $l \circ a$ is an element of $\operatorname{Hom}^{\mathbb{Z}}(B S(\underline{w}), B S(\underline{\mathbf{x}}))$, where $\underline{\mathbf{x}}$ is the reduced expression for $x$ that was fixed in Choices 3.2. Hence, we can write $l \circ a$ in terms of the DLB, and

$$
l \circ a \equiv \sum_{g \in \mathbb{L}_{\underline{w}}(x)} g r_{g} \quad \bmod \mathbb{D L}_{<x}(\underline{w}, \underline{x}),
$$

for some $r_{g} \in R$. Then, the graded cell module $\Delta_{\underline{w}}(x)$ is defined as the graded $\mathbb{R}$-vector space with basis $\mathbb{L}_{\underline{w}}(x)$. The grading on this basis is given by the grading on the leaves, and the $A_{\underline{w}}$-action on $\Delta_{\underline{w}}(x)$ is given by

$$
l \cdot(a \otimes 1)=\sum_{g \in \underline{\mathbb{L}_{\underline{w}}}(x)} g \hat{r_{g}},
$$

for every $l \in \mathbb{L}_{\underline{w}}(x)$ and $a \in \operatorname{End}^{\mathbb{Z}}(B S(\underline{w}))$. Here, $\hat{r_{g}} \in \mathbb{R}$ denotes the reduction modulo $R^{+}$of the scalars, $r_{g}$, that appear in (4.1). The following proposition provides an alternative description of the cell modules.

Proposition 4.3. P14, Lemma 4.6] Assume $\underline{w}$ is a reduced expression for $w \in W$. Then, for all $x \leq w$, we have an isomorphism

$$
\operatorname{Hom}^{\mathbb{Z}}\left(B S(\underline{w}), R_{x}\right) \otimes_{R} \mathbb{R} \cong \Delta_{\underline{w}}(x)
$$

of right $A_{\underline{w}}$-modules, where the $A_{\underline{w}}$-action on $\operatorname{Hom}^{\mathbb{Z}}\left(B S(\underline{w}), R_{x}\right) \otimes_{R} \mathbb{R}$ is given by the composition of morphisms. 
Now we can define a bilinear form, $\langle$,$\rangle , on \Delta_{\underline{w}}(x)$. Let $l_{1}, l_{2} \in \mathbb{L}_{\underline{w}}(x)$. The composition $l_{1} \circ l_{2}^{a}$ belongs to $\operatorname{End}^{\mathbb{Z}}(B S(\underline{\mathbf{x}}))$, and we can expand it in terms of the DLB as

$$
l_{1} \circ l_{2}^{a} \equiv \mathbb{I}_{\underline{\mathbf{x}}} r\left(l_{1}, l_{2}\right) \quad \bmod \mathbb{D L}_{<x}(\underline{x}, \underline{x}),
$$

for some $r\left(l_{1}, l_{2}\right) \in R$. Then, we define the bilinear form on $\Delta_{\underline{w}}(x)$ to be

$$
\left.\left\langle l_{1}, l_{2}\right\rangle=r \widehat{\left(l_{1}, l_{2}\right.}\right) \in \mathbb{R}
$$

where $r \widehat{\left(l_{1}, l_{2}\right)}$ denotes the reduction modulo $R^{+}$of $r\left(l_{1}, l_{2}\right)$. This bilinear form is symmetric, associative and homogeneous, and its radical is defined to be

$$
\operatorname{Rad}\left(\Delta_{\underline{w}}(x)\right)=\left\{l \in \Delta_{\underline{w}}(x) \mid\left\langle l, l^{\prime}\right\rangle=0 \text { for all } l^{\prime} \in \Delta_{\underline{w}}(x)\right\} .
$$

An easy consequence of the associativity and homogeneity of $\langle$,$\rangle is that \operatorname{Rad}\left(\Delta_{\underline{w}}(x)\right)$ is a graded $A_{\underline{w}}$-submodule of $\Delta_{\underline{w}}(x)$. Therefore, we can define the quotient $A_{\underline{w}}$-module $D_{\underline{w}}(x):=\Delta_{\underline{w}}(x) / \operatorname{Rad}\left(\bar{\Delta}_{\underline{w}}(x)\right)$. The modules $\left\{D_{\underline{w}}(x)\right\}_{x \leq w}$ are simple or zero. The bilinear form controls the behavior of the category of Soergel bimodules, as explicitly stated in the following lemma.

Lemma 4.4. Suppose that $\underline{w}$ is a reduced expression for $w \in W$, and define

$$
\Lambda_{0}(\underline{w}):=\left\{x \leq w \mid D_{\underline{w}}(x) \neq 0\right\} .
$$

Then, there exists an isomorphism of graded $(R, R)$-bimodules:

$$
B S(\underline{w})=\bigoplus_{y \in \Lambda_{0}(\underline{w})} \operatorname{dim}_{v} D_{\underline{w}}(y) B_{y}
$$

Proof: A proof for this can be found in [P14, Lemma 4.5]. However, we remark that this proof rephrases the proof of [WI13, Lemma 3.1] using the language of graded cellular algebras.

Given a graded $\mathbb{R}$-vector space $M$ and $k \in \mathbb{Z}$, we let $M\langle k\rangle$ represent the graded $\mathbb{R}$-vector space obtained from $M$ by shifting the grading on $M$, i.e., $M\langle k\rangle_{i}=M_{i-k}$ for all $i \in \mathbb{Z}$.

Remark 4.5. In Section 2.2, the grading shift on graded $(R, R)$-bimodules was defined using round brackets. In the previous paragraph, we have defined the grading shift on graded $\mathbb{R}$-vector spaces by using angled brackets. These two concepts differ only in the direction of the grading shift. In fact, we could have used round or angled brackets to define both concepts. However, we prefer to distinguish them to keep the notation as consistent as possible with both the literature about Soergel bimodules and graded cellular algebras.

The following theorem classifies the set of simple graded $A_{\underline{w}}$-modules.

Theorem 4.6. GL96, Theorem 3.4], [HM10, Theorem 2.10] If $\underline{w}$ is a reduced expression, then the set

$$
\left\{D_{\underline{w}}(x)\langle k\rangle \mid x \in \Lambda_{0}(\underline{w}) \text { and } k \in \mathbb{Z}\right\}
$$

is a complete set of pairwise non-isomorphic graded simple right $A_{\underline{w}}$-modules. 


\subsection{Graded decomposition numbers and KL polynomials}

Suppose $A$ is a graded algebra. If $M$ is a graded $A$-module and $L$ is a graded simple $A$-module, let $[M: L\langle k\rangle]$ be the multiplicity of the simple module $L\langle k\rangle$ as a graded composition factor of $M$, for all $k \in \mathbb{Z}$. The graded decomposition number is defined to be

$$
d_{A}(M, L)=\sum_{k \in \mathbb{Z}}[M: L\langle k\rangle] v^{k} \in \mathbb{Z}\left[v, v^{-1}\right] .
$$

In particular, if $A=A_{\underline{w}}, x \leq w$, and $y \in \Lambda_{0}(\underline{w})$, we will write

$$
d_{\underline{w}}(x, y):=d_{A_{\underline{w}}}\left(\Delta_{\underline{w}}(x), D_{\underline{w}}(y)\right) .
$$

We refer to $\left\{d_{\underline{w}}(x, y) \mid x \leq w\right.$ and $\left.y \in \Lambda_{0}(\underline{w})\right\}$ as the graded decomposition numbers for $A_{\underline{w}}$. The general theory of graded cellular algebras tells us that the graded decomposition numbers for $A_{\underline{w}}$ satisfy the following triangularity property [HM10, Lemma 2.13]:

$$
d_{\underline{w}}(y, y)=1 \text { and } d_{\underline{w}}(x, y)=0 \text { unless } x \leq y,
$$

for every $x \leq w$ and $y \in \Lambda_{0}(\underline{w})$. The following lemma helps relate the graded decomposition numbers for $A_{\underline{w}}$ with the KL polynomials. All the statements in the lemma are well-known, see for example [?, Appendix] for the ungraded case.

Lemma 4.7. Assume $A$ is a graded algebra and $e \in A$ is a homogeneous idempotent. Then the idempotent subalgebra eAe of $A$ is again a graded algebra, and, for any graded right $A$-module $M$, Me has a natural structure of graded right eAe-module. Moreover, if $\left\{L_{\lambda} \mid \lambda \in \Lambda\right\}$ is a complete (up to a degree shift) set of representatives of non-isomorphic classes of graded simple A-modules, then we have the following.

1. The set $\left\{L_{\lambda} e \mid \lambda \in \Lambda\right.$ and $\left.L_{\lambda} e \neq 0\right\}$ is a complete (up to a degree shift) set of representatives of non-isomorphic classes of graded simple eAe-modules.

2. If $M$ is a graded right $A$-module and $L$ is a graded right simple $A$-module such that Le $\neq 0$, then

$$
d_{A}(M, L)=d_{e A e}(M e, L e)
$$

3. Furthermore, if e is a primitive idempotent, then there exists exactly one (up to a degree shift) graded right simple eAe-module. This unique simple module must be of the form Le for some simple graded A-module L.

Proposition 4.8. Let $\underline{w}$ be a reduced expression for $w \in W$. Then

$$
d_{\underline{w}}(x, y)=h_{x, y}
$$

for all $x \leq w$ and $y \in \Lambda_{0}(\underline{w})$.

Proof: First, we recall from Theorem 2.6 that $h_{x, y}=\operatorname{dim}_{v}\left(\operatorname{Hom}^{\mathbb{Z}}\left(B_{y}, R_{x}\right) \otimes_{R} \mathbb{R}\right)$. In addition, Lemma 4.4 states that, for all $y \in \Lambda_{0}(\underline{w})$, we can choose a primitive idempotent, $\epsilon_{y}^{k} \in \operatorname{End}^{\mathbb{Z}}(B S(\underline{w}))$, whose image is isomorphic to $B_{y}(k)$ for some $k \in \mathbb{Z}$. Reduction modulo $R^{+}$of $\epsilon_{y}^{k}$, which we simply denote using $e$, is again a primitive idempotent in $A_{\underline{w}}$. According to Lemma 4.7, the idempotent subalgebra $e A_{\underline{w}} e$ is a graded algebra with a unique (up to a degree shift) simple graded right module. Furthermore, by Theorem 4.6 and Lemma 4.7, we know that the unique simple 
graded $e A_{\underline{w}} e$-module is of the form $D_{\underline{w}}(z) e \neq 0$, for some $z \in \Lambda_{0}(\underline{w})$. We claim that $z=y$. To prove this claim, for $x \leq w$, we have

$$
\begin{aligned}
v^{k} \operatorname{dim}_{v}\left(\operatorname{Hom}^{\mathbb{Z}}\left(B_{y}, R_{x}\right) \otimes_{R} \mathbb{R}\right) & =\operatorname{dim}_{v}\left(\operatorname{Hom}^{\mathbb{Z}}\left(B_{y}(k), R_{x}\right) \otimes_{R} \mathbb{R}\right) \\
& =\operatorname{dim}_{v}\left(\left(\operatorname{Hom}^{\mathbb{Z}}\left(B S(\underline{w}), R_{x}\right) \otimes_{R} \mathbb{R}\right) e\right) \\
& =\operatorname{dim}_{v}\left(\Delta_{\underline{w}}(x) e\right) \\
& =d_{e A_{\underline{w}}}\left(\Delta_{\underline{w}}(x) e, D_{\underline{w}}(z) e\right) \operatorname{dim}_{v}\left(D_{\underline{w}}(z) e\right) \\
& =d_{\underline{w}}(x, z) \underline{\operatorname{dim}_{v}}\left(D_{\underline{w}}(z) e\right) .
\end{aligned}
$$

Here, the second equation is a result of the definition of $e$, the third equation is a consequence of Proposition 4.3 , the fourth equation follows from the fact that $D_{\underline{w}}(z) e$ is the unique simple $e A_{\underline{w}} e$-module, and the last equation is obtained using Lemma 4.7, Substituting $x=y$ into (4.14) gives us

$$
v^{k} \operatorname{dim}_{v}\left(\operatorname{Hom}^{\mathbb{Z}}\left(B_{y}, R_{y}\right) \otimes_{R} \mathbb{R}\right)=d_{\underline{w}}(y, z) \operatorname{dim}_{v}\left(D_{\underline{w}}(z) e\right) .
$$

Because $\operatorname{dim}_{v}\left(\operatorname{Hom}^{\mathbb{Z}}\left(B_{y}, R_{y}\right) \otimes_{R} \mathbb{R}\right)=h_{y, y}=1$, the right side of (4.15) is not zero. Therefore, $d_{\underline{w}}(y, z) \neq 0$, and applying (4.11) gives us $y \leq z$. On the other hand, substituting $x=z$ into (4.14) provides

$$
v^{k} \operatorname{dim}_{v}\left(\operatorname{Hom}^{\mathbb{Z}}\left(B_{y}, R_{z}\right) \otimes_{R} \mathbb{R}\right)=d_{\underline{w}}(z, z) \operatorname{dim}_{v}\left(D_{\underline{w}}(z) e\right) .
$$

Again, by (4.11) we know that $d_{\underline{w}}(z, z)=1$. Thus, we conclude that $h_{z, y}=\operatorname{dim}_{v}\left(\operatorname{Hom}^{\mathbb{Z}}\left(B_{y}, R_{z}\right) \otimes_{R} \mathbb{R}\right) \neq 0$, and $y \geq z$. This proves our claim.

Furthermore, if we substitute $y=z$ into (4.14), we obtain

$$
v^{k} \operatorname{dim}_{v}\left(\operatorname{Hom}^{\mathbb{Z}}\left(B_{y}, R_{x}\right) \otimes_{R} \mathbb{R}\right)=d_{\underline{w}}(y, x) \operatorname{dim}_{v}\left(D_{\underline{w}}(y) e\right) .
$$

Finally, substituting $x=y$ into (4.17) provides $\operatorname{dim}_{v}\left(D_{\underline{w}}(y) e\right)=v^{k}$, and cancelling these terms in (4.17) produces the desired equality.

\section{BRANCHING RULES}

In order to proceed, we fix from now on a reduced expression $\underline{w}=s_{1} \ldots s_{k}$ of $w \in W$. In addition, in order to simplify the notation, we refer to $s_{1}$ simply as $s$. Define $x^{\prime}=s x \in W$, for all $x \in W$. In particular, we have $w^{\prime}=s w$. From now on we fix the reduced expression $\underline{w^{\prime}}=s_{2} \ldots s_{k}$ for $w^{\prime}$. By definition of $A_{\underline{w}}$ and $A_{\underline{w^{\prime}}}$ it is clear that there exists an injective $\mathbb{R}$-algebra homomorphism from $A_{\underline{w^{\prime}}}$ to $A_{\underline{w}}$, which sends an element $a \underline{\epsilon} A_{\underline{w^{\prime}}}$ to $\mathbb{I}_{s} \otimes a \in A_{\underline{w}}$. This embedding allows us to view $A_{\underline{w^{\prime}}}$ as a subalgebra of $A_{\underline{w}}$. Accordingly, any $A_{\underline{w}}$-module $M$ can be considered as an $A_{\underline{w^{\prime}}}$-module by restriction. We denote the restricted module by $\operatorname{Res}(M)$. In this section, we obtain graded branching rules for graded cell modules and graded simple modules of $A_{\underline{w}}$. We end this section by showing how to utilize these graded branching rules to recover a normalized version of (1.3).

\subsection{Branching rules for cell modules}

Consider the expression $\underline{w^{\prime}}$ and its corresponding tree $\mathbb{T}_{\underline{w^{\prime}}}$. Recall that in order to begin the construction of $\mathbb{T}_{\underline{w}^{\prime}}$ we must fix some data (see Choices 3.2). In particular, the reduced expression $\underline{\mathbf{x}}$ must be fixed, for all $x \in W$. There is no loss of generality in assuming that $s$ appears on the left side of $\underline{\mathbf{x}}$, for every $x$ with $x^{\prime}<x$. After the reduced expression has been fixed for every element $x$ with $x^{\prime}<x$, we can fix reduced expressions $\mathbf{y}$ for every element $y \in W$, where $y^{\prime}>y$, according to the following rule: $\underline{\mathbf{y}}$ is obtained from $\underline{\mathbf{y}}^{\prime}$ by deleting the $s$ located to the left of $\underline{\mathbf{y}^{\prime}}$. It should be noted that $\underline{\mathbf{y}}^{\prime}$ has already been fixed since $s y^{\prime}<y^{\prime}$.

The consequence of these choices is that the tree $\mathbb{T}_{\underline{w}}$ can be obtained directly from $\mathbb{T}_{\underline{w^{\prime}}}$. Concretely, each leaf $l \in \mathbb{L}_{\underline{w^{\prime}}}(x)$ gives rise to two leaves in $\mathbb{T}_{\underline{w}}$, according to $\overline{x^{\prime}}<x$ or $x^{\prime}>x$, which is illustrated in Figure 4. Although 

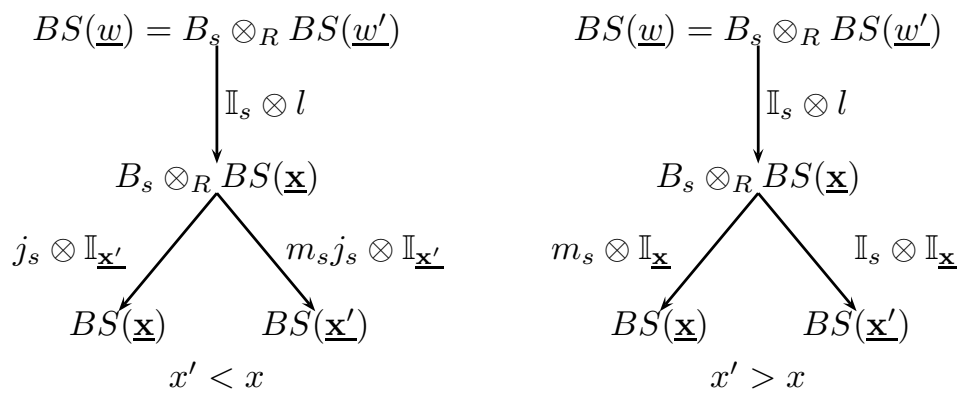

Figure 4: Constructing leaves from $\mathbb{T}_{\underline{w^{\prime}}}$ to $\mathbb{T}_{\underline{w}}$

this could be considered a direct consequence of the construction of $\mathbb{T}_{\underline{w}}$, we note the absence of morphisms of type $F($, , ) in Figure 4 , which results from the previously realized choices.

Lemma 5.1. Given $x \leq w$, the set $\mathbb{L}_{\underline{w}}(x)$ can be partitioned into two disjoint sets, $Y_{\underline{w}, 1}(x)$ and $Y_{\underline{w}, 2}(x)$, which are defined as follows.

$$
\begin{aligned}
& Y_{\underline{w}, 1}(x)=\left\{\begin{array}{l}
\left\{\left(\mathbb{I}_{s} \otimes l\right) \mid l \in \mathbb{L}_{\underline{w^{\prime}}}\left(x^{\prime}\right)\right\}, \quad \text { if } x^{\prime}<x ; \\
\left\{\left(m_{s} \otimes l\right) \mid l \in \mathbb{L}_{\underline{w^{\prime}}}(x)\right\}, \quad \text { if } x^{\prime}>x
\end{array}\right. \\
& Y_{\underline{w}, 2}(x)= \begin{cases}\left\{\left(j_{s} \otimes \mathbb{I}_{\underline{\mathbf{x}^{\prime}}}\right)\left(\mathbb{I}_{s} \otimes l\right) \mid l \in \mathbb{L}_{\underline{w^{\prime}}}(x)\right\}, & \text { if } x^{\prime}<x ; \\
\left\{\left(m_{s} j_{s} \otimes \mathbb{I}_{\underline{\mathbf{x}^{\prime}}}\right)\left(\mathbb{I}_{s} \otimes l\right) \mid l \in \mathbb{L}_{\underline{w^{\prime}}}\left(x^{\prime}\right)\right\}, & \text { if } x^{\prime}>x\end{cases}
\end{aligned}
$$

Proof: The reason for this is clear given the construction of the leaves in $\mathbb{T}_{\underline{w}}$ from the leaves in $\mathbb{T}_{\underline{w^{\prime}}}$, which is provided in Figure 4

The branching rules for $\Delta_{\underline{w}}(x)$ can be classified into two cases: $x^{\prime}<x$ and $x^{\prime}>x$. In the following theorem, we assume $x^{\prime}<x$.

Theorem 5.2. Suppose $x \leq w$ is such that $x^{\prime}<x$. Then, there exists a short exact sequence of graded $A_{\underline{w^{\prime}}}$-modules,

$$
0 \longrightarrow \operatorname{Hom}^{\mathbb{Z}}\left(B S\left(\underline{w^{\prime}}\right), R_{x^{\prime}}\right) \otimes_{R} \mathbb{R} \stackrel{\phi_{1}}{\longrightarrow} \operatorname{Res}\left(\operatorname{Hom}^{\mathbb{Z}}\left(B S(\underline{w}), R_{x}\right) \otimes_{R} \mathbb{R}\right) \stackrel{\phi_{2}}{\longrightarrow}\left(\operatorname{Hom}^{\mathbb{Z}}\left(B S\left(\underline{w^{\prime}}\right), R_{x}\right) \otimes_{R} \mathbb{R}\right)\langle-1\rangle \longrightarrow 0
$$

in which $\phi_{1}(f)=\beta_{s} \otimes f$ and $\phi_{2}(f)=f\left(e_{s} \otimes \mathbb{I}_{\underline{w^{\prime}}}\right)$.

Proof: Assume $a \in A_{\underline{w^{\prime}}}$. Then, for all $f \in \operatorname{Hom}^{\mathbb{Z}}\left(B S\left(\underline{w^{\prime}}\right), R_{x^{\prime}}\right) \otimes_{R} \mathbb{R}$, we have

$$
\phi_{1}(f a)=\beta_{s} \otimes(f a)=\left(\beta_{s} \otimes f\right)\left(\mathbb{I}_{s} \otimes a\right)=\phi_{1}(f)\left(\mathbb{I}_{s} \otimes a\right),
$$

proving that $\phi_{1}$ is an $A_{\underline{w^{\prime}}}$-algebra homomorphism. Similarly, we have

$$
\phi_{2}\left(f\left(\mathbb{I}_{s} \otimes a\right)\right)=f\left(\mathbb{I}_{s} \otimes a\right)\left(e_{s} \otimes \mathbb{I}_{\underline{w^{\prime}}}\right)=f\left(e_{s} \otimes \mathbb{I}_{\underline{w^{\prime}}}\right) a=\phi_{2}(f) a,
$$

for every $f \in \operatorname{Hom}^{\mathbb{Z}}\left(B S(\underline{w}), R_{x}\right) \otimes_{R} \mathbb{R}$. Hence, $\phi_{2}$ is also an $A_{\underline{w^{\prime}}}$-algebra homomorphism. Furthermore, we note that $\phi_{1}$ preserves the grading, and $\phi_{2}$ increases the grading by 1 because $\operatorname{deg}\left(e_{s}\right)=1$. In summary, both $\phi_{1}$ and $\phi_{2}$ are homogeneous $A_{\underline{w^{\prime}}}$-algebra homomorphisms of degree 0 and 1 , respectively. 
DAVID PLAZA

Now, we are going to prove that $\phi_{1}$ and $\phi_{2}$ are injective and surjective, respectively. Proposition 3.5 ensures that $\mathbb{L}_{\underline{w}}^{\beta}(x)$ is an $R$-basis of $\operatorname{Hom}^{\mathbb{Z}}\left(B S(\underline{w}), R_{x}\right)$. Therefore, the image of $\mathbb{L}_{\underline{w}}^{\beta}(x)$ under reduction modulo $R^{+}$is an $\mathbb{R}$-basis of $\operatorname{Hom}^{\mathbb{Z}}\left(B S(\underline{w}), R_{x}\right) \otimes_{R} \mathbb{R}$. If we relax the notation, we can also consider $\mathbb{L}_{\underline{w}}^{\beta}(x)$ to be an $\mathbb{R}$-basis of $\operatorname{Hom}^{\mathbb{Z}}\left(B S(\underline{w}), R_{x}\right) \otimes_{R} \mathbb{R}$. Lemma 5.1 implies that, given $x^{\prime}<x, \mathbb{L}_{\underline{w}}^{\beta}(x)$ can be partitioned into two disjoint sets:

$$
\begin{aligned}
& Y_{\underline{w}, 1}^{\beta}(x):=\left\{\beta_{\underline{\mathbf{x}}} \circ\left(\mathbb{I}_{s} \otimes l\right) \mid l \in \mathbb{L}_{\underline{w^{\prime}}}\left(x^{\prime}\right)\right\}, \text { and } \\
& Y_{\underline{w}, 2}^{\beta}(x):=\left\{\beta_{\underline{\mathbf{x}}} \circ\left(j_{s} \otimes \mathbb{I}_{\underline{\mathbf{x}^{\prime}}}\right)\left(\mathbb{I}_{s} \otimes l\right) \mid l \in \mathbb{L}_{\underline{w^{\prime}}}(x)\right\} .
\end{aligned}
$$

For all $l \in \mathbb{L}_{\underline{w^{\prime}}}\left(x^{\prime}\right)$, we have

$$
\begin{aligned}
\phi_{1}\left(\beta_{\underline{\mathbf{x}^{\prime}}} \circ l\right) & =\beta_{s} \otimes\left(\beta_{x^{\prime}} \circ l\right) \\
& =\left(\beta_{s} \otimes \beta_{\mathbf{x}^{\prime}}\right)\left(\mathbb{I}_{s} \otimes l\right) \\
& =\beta_{\underline{\mathbf{x}}} \circ\left(\mathbb{I}_{s} \otimes l\right) .
\end{aligned}
$$

Therefore, $\phi_{1}\left(\mathbb{L}_{\underline{w^{\prime}}}^{\beta}\left(x^{\prime}\right)\right)=Y_{\underline{w}, 1}^{\beta}(x)$, and $\phi_{1}$ is injective. Similarly, for $l \in \mathbb{L}_{\underline{w^{\prime}}}(x)$ we have

$$
\begin{aligned}
\phi_{2}\left(\beta_{\underline{\mathbf{x}}} \circ\left(j_{s} \otimes \mathbb{I}_{\underline{\mathbf{x}^{\prime}}}\right)\left(\mathbb{I}_{s} \otimes l\right)\right) & =\beta_{\underline{\mathbf{x}}} \circ\left(j_{s} \otimes \mathbb{I}_{\underline{\mathbf{x}^{\prime}}}\right)\left(\mathbb{I}_{s} \otimes l\right)\left(e_{s} \otimes \mathbb{I}_{\underline{w^{\prime}}}\right) \\
& =\beta_{\underline{\mathbf{x}}} \circ\left(j_{s} \otimes \mathbb{I}_{\underline{\mathbf{x}^{\prime}}}\right)\left(e_{s} \otimes l\right) \\
& =\beta_{\underline{\mathbf{x}}} \circ l
\end{aligned}
$$

because $j_{s}\left(e_{s} \otimes \mathbb{I}_{s}\right)=\mathbb{I}_{s}$. Hence, $\phi_{2}\left(Y_{\underline{w}, 2}^{\beta}(x)\right)=\mathbb{L}_{\underline{w^{\prime}}}^{\beta}(x)$, and $\phi_{2}$ is surjective.

Finally, in order to complete the proof we need to show that $\operatorname{Im}\left(\phi_{1}\right)=\operatorname{ker}\left(\phi_{2}\right)$. For each $l \in \mathbb{L}_{\underline{w^{\prime}}}\left(x^{\prime}\right)$, we have

$$
\phi_{2} \phi_{1}\left(\beta_{\underline{\mathbf{x}^{\prime}}} \circ l\right)=\phi_{2}\left(\beta_{\underline{\mathbf{x}}} \circ\left(\mathbb{I}_{s} \otimes l\right)\right)=\beta_{\underline{\mathbf{x}}}\left(\mathbb{I}_{s} \otimes l\right)\left(e_{s} \otimes \mathbb{I}_{w^{\prime}}\right)=\beta_{\underline{\mathbf{x}}}\left(e_{s} \otimes \mathbb{I}_{\mathbf{x}^{\prime}}\right) l=0,
$$

because $\beta_{\underline{\mathbf{x}}}\left(e_{s} \otimes \mathbb{I}_{\underline{\mathbf{x}^{\prime}}}\right) \in \operatorname{Hom}^{\mathbb{Z}}\left(B S\left(\underline{\mathbf{x}^{\prime}}\right), R_{x}\right) \otimes_{R} \mathbb{R}=0$, according to Corollary 3.6. Equation (5.5) implies that $\operatorname{Im}\left(\phi_{1}\right) \subset \operatorname{ker}\left(\phi_{2}\right)$. Moreover, an easy counting argument reveals that $\operatorname{Im}\left(\phi_{1}\right)=\operatorname{ker}\left(\phi_{2}\right)$, completing the proof.

Corollary 5.3. Assume $x \leq w$ is such that $x^{\prime}<x$. Then there exists a short exact sequence of graded $A_{\underline{w^{\prime}}}$-modules

$$
0 \longrightarrow \Delta_{\underline{w^{\prime}}}\left(x^{\prime}\right) \longrightarrow \operatorname{Res}\left(\Delta_{\underline{w}}(x)\right) \longrightarrow \Delta_{\underline{w^{\prime}}}(x)\langle-1\rangle \longrightarrow 0 .
$$

Proof: This is a direct consequence of Proposition 4.3 and Theorem 5.2 .

Analogous results for Theorem 5.2 and Corollary 5.3 also exist for the case in which $x^{\prime}>x$.

Theorem 5.4. If $x \leq w$ is given such that $x^{\prime}>x$, then there exists a graded short exact sequence of $A_{\underline{w^{\prime}}}$-modules,

$$
0 \longrightarrow\left(\operatorname{Hom}^{\mathbb{Z}}\left(B S\left(\underline{w^{\prime}}\right), R_{x}\right) \otimes_{R} \mathbb{R}\right)\langle 1\rangle \stackrel{\phi_{1}}{\longrightarrow} \operatorname{Res}\left(\operatorname{Hom}^{\mathbb{Z}}\left(B S(\underline{w}), R_{x}\right) \otimes_{R} \mathbb{R}\right) \stackrel{\phi_{2}}{\longrightarrow} \operatorname{Hom}^{\mathbb{Z}}\left(B S\left(\underline{w^{\prime}}\right), R_{x^{\prime}}\right) \otimes_{R} \mathbb{R} \longrightarrow 0,
$$

where $\phi_{1}(f)=m_{s} \otimes f$ and $\phi_{2}(f)=\left(\beta_{s} \otimes f\right)\left(p_{s} e_{s} \otimes \mathbb{I}_{\underline{w^{\prime}}}\right)$. Equivalently, there exists a graded short exact sequence of A $\underline{\underline{w^{\prime}}}$-modules:

$$
0 \longrightarrow \Delta_{\underline{w^{\prime}}}(x)\langle 1\rangle \longrightarrow \operatorname{Res}\left(\Delta_{\underline{w}}(x)\right) \longrightarrow \Delta_{\underline{w^{\prime}}}\left(x^{\prime}\right) \longrightarrow 0 .
$$

Proof: This proof is analogous to that of Theorem 5.2

In order to continue, it would be useful to rephrase the branching rules in terms of Grothendieck groups. Given a reduced expression $\underline{w}$, the Grothendieck group $\mathcal{G}_{\underline{w}}$ of $A_{\underline{w}}$ is the $\mathbb{Z}\left[v, v^{-1}\right]$-module generated by symbols $[M]$, in which $M$ runs over all isomorphism classes of graded $A_{\underline{w}}$-modules, together with the following relations:

1. $[M\langle k\rangle]=v^{k}[M]$, for every graded right $A_{\underline{w}}$-module $M$ and every $k \in \mathbb{Z}$; 
2. $[M]=\left[M^{\prime}\right]+\left[M^{\prime \prime}\right]$, if there exists a (graded) short exact sequence $0 \rightarrow M^{\prime} \rightarrow M \rightarrow M^{\prime \prime} \rightarrow 0$ of graded right $A_{\underline{w}}$-modules.

Thus, Theorem 4.6 reveals that $\mathcal{G}_{\underline{w}}$ is free as a $\mathbb{Z}\left[v, v^{-1}\right]$-module with a basis given by $\left\{\left[D_{\underline{w}}(x)\right] \mid x \in \Lambda_{0}(\underline{w})\right\}$. Using this terminology, the graded short exact sequences in Theorem 5.2 and Theorem 5.4 can be rephrased as follows.

Theorem 5.5. There exists a homomorphism of $\mathbb{Z}\left[v, v^{-1}\right]$-modules Res : $\mathcal{G}_{\underline{w}} \rightarrow \mathcal{G}_{\underline{w^{\prime}}}$ determined by

$$
\operatorname{Res}([M])=[\operatorname{Res}(M)]
$$

for every right graded $A_{\underline{w}}$-module $M$. The image of the class of cell module $\Delta_{\underline{w}}(x)$ under this homomorphism is

$$
\operatorname{Res}\left(\left[\Delta_{\underline{w}}(x)\right]\right)=\left\{\begin{aligned}
v^{-1}\left[\Delta_{\underline{w^{\prime}}}(x)\right]+\left[\Delta_{\underline{w^{\prime}}}\left(x^{\prime}\right)\right], & \text { if } x^{\prime}<x \\
v\left[\Delta_{\underline{w^{\prime}}}(x)\right]+\left[\Delta_{\underline{w^{\prime}}}\left(x^{\prime}\right)\right], & \text { if } x^{\prime}>x
\end{aligned}\right.
$$

\subsection{Branching rules for simple modules}

In this section, we obtain graded branching rules for simple $A_{\underline{w}}$-modules. In other terms, we calculate the image of the class $\left[D_{\underline{w}}(x)\right]$ under the action of the homomorphism Res, for all $x \in \Lambda_{0}(\underline{w})$. In order to do this, we define a $\mathbb{Z}\left[v, v^{-1}\right]$-homomorphism $\rho$ from $\mathcal{G}_{\underline{w}}$ to $\mathcal{G}_{\underline{w^{\prime}}}$ and prove that $\rho$ coincides with Res. Given $u \in \Lambda_{0}\left(\underline{w^{\prime}}\right)$, there exist polynomials $h_{s, u}^{x} \in \mathbb{Z}\left[v, v^{-1}\right]$ such that

$$
B_{s} \otimes B_{u} \cong \bigoplus_{x \in \Lambda_{0}(\underline{w})} h_{s, u}^{x} B_{x}
$$

In principle, the sum on the right side of (5.11) should run over $W$. However, Lemma 4.4 guarantees that if $x \notin \Lambda_{0}(\underline{w})$, then the polynomials $h_{s, u}^{x}$ equal zero.

Using the polynomials $h_{s, u}^{x}$, we can define a $\mathbb{Z}\left[v, v^{-1}\right]$-homomorphism $\rho: \mathcal{G}_{\underline{w}} \rightarrow \mathcal{G}_{\underline{w^{\prime}}}$ determined in the basis $\left\{\left[D_{\underline{w}}(x)\right] \mid x \in \Lambda_{0}(\underline{w})\right\}$ of $\mathcal{G}_{\underline{w}}$ by

$$
\rho\left(\left[D_{\underline{w}}(x)\right]\right)=\sum_{u \in \Lambda_{0}\left(\underline{w^{\prime}}\right)} h_{s, u}^{x}\left[D_{\underline{w^{\prime}}}(u)\right]
$$

for every $x \in \Lambda_{0}(\underline{w})$. In order to prove that $\rho=$ Res, we need the following lemmas.

Lemma 5.6. Given any $z \leq w$ and any $u \in \Lambda_{0}\left(\underline{w^{\prime}}\right)$, we have

$$
d_{A_{\underline{w^{\prime}}}}\left(\operatorname{Res}\left(\Delta_{\underline{w}}(z)\right), D_{\underline{w^{\prime}}}(u)\right)=\sum_{x \in \Lambda_{0}(\underline{w})} h_{s, u}^{x} d_{\underline{w}}(z, x) .
$$

Proof: Assuming $u \in \Lambda_{0}\left(\underline{w^{\prime}}\right)$, we can choose a primitive idempotent, $\epsilon_{u}^{k} \in \operatorname{End}^{\mathbb{Z}}\left(B S\left(\underline{w^{\prime}}\right)\right)$, whose image is isomorphic to $B_{u}(k)$, for some $k \in \mathbb{Z}$. Reduction modulo $R^{+}$of $\epsilon_{u}^{k}$, which we denote as $e \in A_{\underline{w^{\prime}}}$, is a primitive idempotent, and from Lemma 4.7 we know the algebra $e A_{\underline{w^{\prime}}} e$ has a unique (up to a degree shift) graded simple module. In the proof of Proposition 4.8, we have already proven that the unique simple module of $e A_{\underline{w^{\prime}}} e$ is $D_{\underline{w^{\prime}}}(u) e$. Furthermore, we can also prove that $\operatorname{dim}_{v} D_{\underline{w^{\prime}}}(u) e=v^{k}$. Thus, we have the following chain of equations. 


$$
\begin{aligned}
d_{A_{\underline{w^{\prime}}}}\left(\operatorname{Res}\left(\Delta_{\underline{w}}(z)\right), D_{\underline{w^{\prime}}}(u)\right) v^{k} & =d_{A_{\underline{w^{\prime}}}}\left(\operatorname{Res}\left(\Delta_{\underline{w}}(z)\right), D_{\underline{w^{\prime}}}(u)\right) \operatorname{dim}_{v} D_{\underline{w^{\prime}}}(u) e \\
& =d_{e A_{\underline{w}^{\prime}}}\left(\operatorname{Res}\left(\Delta_{\underline{w}}(z)\right)\left(\mathbb{I}_{s} \otimes e\right), D_{\underline{w^{\prime}}}(u) e\right) \operatorname{dim}_{v} D_{\underline{w^{\prime}}}(u) e \\
& =\operatorname{dim}_{v} \Delta_{\underline{w}}(z)\left(\mathbb{I}_{s} \otimes e\right) \\
& =\operatorname{dim}_{v}\left(\operatorname{Hom}^{\mathbb{Z}}\left(B S(\underline{w}), R_{z}\right) \otimes_{R} \mathbb{R}\right)\left(\mathbb{I}_{s} \otimes e\right) \\
& =v^{k} \operatorname{dim}_{v}\left(\operatorname{Hom}^{\mathbb{Z}}\left(B_{s} \otimes B_{u}, R_{z}\right) \otimes_{R} \mathbb{R}\right) \\
& =v^{k} \sum_{x \in \Lambda_{0}(\underline{w})} h_{s, u}^{x} \operatorname{dim}_{v}\left(\operatorname{Hom}^{\mathbb{Z}}\left(B_{x}, R_{z}\right) \otimes_{R} \mathbb{R}\right) \\
& =v^{k} \sum_{x \in \Lambda_{0}(\underline{w})} h_{s, u}^{x} d_{\underline{w}}(z, x) .
\end{aligned}
$$

Here, we obtain (5.14) because: $\operatorname{dim}_{v} D_{\underline{w^{\prime}}}(u) e=v^{k}$; (5.15) follows from Lemma 4.7. (5.16) applies the fact that $D_{\underline{w^{\prime}}}(u) e$ is the unique (up to a degree shift) simple $e A_{\underline{w^{\prime}}} e$-module; Proposition4.3 implies (5.17); the definition of $e$ justifies (5.18); (5.19) is a consequence of (5.11); and (5.20) follows from Theorem 2.6] and Proposition 4.8. Finally, multiplying the chain of equations by $v^{-k}$, we achieve the desired equality.

Lemma 5.7. Given a graded right $A_{\underline{w}}$-module $M$, we have the following equation in $\mathcal{G}_{\underline{w}}$ :

$$
[M]=\sum_{x \in \Lambda_{0}(\underline{w})} d_{A_{\underline{w}}}\left(M, D_{\underline{w}}(x)\right)\left[D_{\underline{w}}(x)\right] .
$$

Likewise, the same is true if we replace $\underline{w}$ with $\underline{w^{\prime}}$.

Proof: This is a direct consequence of the definitions of graded decomposition numbers and Grothendieck groups.

Theorem 5.8. The homomorphisms Res and $\rho$ coincide.

Proof: First, according to the triangularity property in (4.11), we know that $\left\{\left[\Delta_{\underline{w}}(x)\right]\right\}_{x \in \Lambda_{0}(\underline{w})}$ is also a $\mathbb{Z}\left[v, v^{-1}\right]$ basis of $\mathcal{G}_{\underline{w}}$. Therefore, in order to prove the theorem, it is sufficient to show that

$$
\operatorname{Res}\left(\left[\Delta_{\underline{w}}(x)\right]\right)=\rho\left(\left[\Delta_{\underline{w}}(x)\right]\right),
$$

for all $x \in \Lambda_{0}(\underline{w})$. More generally, we will prove that (5.22) holds for all $x \leq w$. According to Lemma 5.7 given $x \leq w$, we have

$$
\left[\Delta_{\underline{w}}(x)\right]=\sum_{y \in \Lambda_{0}(\underline{w})} d_{\underline{w}}(x, y)\left[D_{\underline{w}}(y)\right] .
$$

Thus, applying the map $\rho$ to (5.23) and using the definition of $\rho$ provided in (5.12) gives us

$$
\rho\left(\left[\Delta_{\underline{w}}(x)\right]\right)=\sum_{u \in \Lambda_{0}\left(\underline{w^{\prime}}\right)}\left(\sum_{y \in \Lambda_{0}(\underline{w})} h_{s, u}^{y} d_{\underline{w}}(x, y)\right)\left[D_{\underline{w^{\prime}}}(u)\right],
$$

for every $x \leq w$. On the other hand, the combination of Lemmas 5.6 and 5.7 implies that the right side of (5.24) equals $\operatorname{Res}\left(\left[\Delta_{\underline{w}}(x)\right]\right)$, for all $x \leq w$. This completes the proof.

Corollary 5.9. If $x \in \Lambda_{0}(\underline{w})$, then the restriction of the simple module $D_{\underline{w}}(x)$ is given by

$$
\operatorname{Res}\left(\left[D_{\underline{w}}(x)\right]\right)=\sum_{u \in \Lambda_{0}\left(\underline{w^{\prime}}\right)} h_{s, u}^{x}\left[D_{\underline{w^{\prime}}}(u)\right] .
$$




\subsection{Categorifying a recursive formula for KL polynomials}

In this subsection, we use the branching rules for graded cell modules and graded simple modules in order to obtain (2.10). In other words, we use the categories of graded finite-dimensional $A_{\underline{w}}$-modules and $A_{\underline{w^{\prime}}}$-modules (and, therefore, the category of Soergel bimodules) in order to recover (2.10). In short, we categorify (2.10). Throughout this subsection, we maintain the notation provided in this section's introduction.

In order to categorify (2.10), we need the following lemma. First, however, we recall that $\mu\left(z, w^{\prime}\right)$ denotes the linear coefficient of the Kazhdan-Lusztig polynomial $h_{z, w^{\prime}}$, for all $z \in W$ (see section 2.1).

Lemma 5.10. For all $z<w$, we have

$$
h_{s, w^{\prime}}^{z}=\mu\left(z, w^{\prime}\right)
$$

Furthermore, we also have $h_{s, w^{\prime}}^{w}=1$.

Proof: First, we recall that the polynomials $h_{s, w^{\prime}}^{z}$ were defined by the isomorphism

$$
B_{s} \otimes_{R} B_{w^{\prime}} \cong \bigoplus_{z \in \Lambda_{0}(\underline{w})} h_{s, w^{\prime}}^{z} B_{z} .
$$

According to Soergel's conjecture, this isomorphism implies that

$$
\underline{H}_{s} \underline{H}_{w^{\prime}}=\sum_{z \in \Lambda_{0}(\underline{w})} h_{s, w^{\prime}}^{z} \underline{H}_{z}
$$

at the Hecke-algebra level. On the other hand, (2.8) tells us that

$$
\underline{H}_{s} \underline{H}_{w^{\prime}}=\underline{H}_{w}+\sum_{z<w} \mu\left(z, w^{\prime}\right) \underline{H}_{z} .
$$

Therefore, the lemma is proved by comparing the coefficients in (5.28) and (5.29).

According to Lemma 5.7, in $\mathcal{G}_{\underline{w}}$, we have

$$
\left[\Delta_{\underline{w}}(x)\right]=\sum_{z \in \Lambda_{0}(\underline{w})} d_{\underline{w}}(x, z)\left[D_{\underline{w}}(z)\right]
$$

for all $x \leq w$. Hence, if we apply the homomorphism Res to (5.30), and if we use Theorem [5.5] and Corollary [5.9] then we know that, in $\mathcal{G}_{\underline{w^{\prime}}}$,

$$
\sum_{u \in \Lambda_{0}\left(\underline{w^{\prime}}\right)}\left(\sum_{z \in \Lambda_{0}(\underline{w})} h_{s, u}^{z} d_{\underline{w}}(x, z)\right)\left[D_{\underline{w^{\prime}}}(u)\right]=\left\{\begin{aligned}
v^{-1}\left[\Delta_{\underline{w^{\prime}}}(x)\right]+\left[\Delta_{\underline{w^{\prime}}}\left(x^{\prime}\right)\right], & \text { if } x^{\prime}<x ; \\
v\left[\Delta_{\underline{w^{\prime}}}(x)\right]+\left[\Delta_{\underline{w^{\prime}}}\left(x^{\prime}\right)\right], & \text { if } x^{\prime}>x .
\end{aligned}\right.
$$

Furthermore, if we recall that $\left\{\left[D_{\underline{w^{\prime}}}(u)\right]\right\}_{u \in \Lambda_{0}\left(\underline{w^{\prime}}\right)}$ is a $\mathbb{Z}\left[v, v^{-1}\right]$-basis of $\mathcal{G}_{\underline{w^{\prime}}}$, then (5.31) provides a family of equations in $\mathbb{Z}\left[v, v^{-1}\right]$ (one for each element in $\Lambda_{0}\left(\underline{w^{\prime}}\right)$ ). In general, there is no simple, direct method for determining $\Lambda_{0}\left(\underline{w^{\prime}}\right)$. However, it is easy to conclude that $w^{\prime} \in \Lambda_{0}\left(\underline{w^{\prime}}\right)$. The equation obtained by taking the coefficient of $\left[D_{\underline{w^{\prime}}}\left(w^{\prime}\right)\right]$ in (5.31) is

$$
\sum_{z \in \Lambda_{0}(\underline{w})} h_{s, w^{\prime}}^{z} d_{\underline{w}}(x, z)=\left\{\begin{aligned}
v^{-1} d_{\underline{w^{\prime}}}\left(x, w^{\prime}\right)+d_{\underline{w^{\prime}}}\left(x^{\prime}, w^{\prime}\right), & \text { if } x^{\prime}<x \\
v d_{\underline{w^{\prime}}}\left(x, w^{\prime}\right)+d_{\underline{w^{\prime}}}\left(x^{\prime}, w^{\prime}\right), & \text { if } x^{\prime}>x
\end{aligned}\right.
$$


Consequently, we can apply Proposition 4.8 and Lemma 5.10 to rewrite (5.32) as

$$
h_{x, w}+\sum_{\substack{z \in \Lambda_{0}(\underline{w}) \\
z \neq w}} \mu\left(z, w^{\prime}\right) h_{x, z}=\left\{\begin{array}{cl}
v^{-1} h_{x, w^{\prime}}+h_{x^{\prime}, w^{\prime}}, & \text { if } x^{\prime}<x ; \\
v h_{x, w^{\prime}}+h_{x^{\prime}, w^{\prime}}, & \text { if } x^{\prime}>x .
\end{array}\right.
$$

Reordering the terms in this equation gives us

$$
h_{x, w}=\left\{\begin{array}{cc}
v^{-1} h_{x, w^{\prime}}+h_{x^{\prime}, w^{\prime}}-\sum_{\substack{z \in \Lambda_{0}(\underline{w}) \\
z \neq w}} \mu\left(z, w^{\prime}\right) h_{x, z}, & \text { if } x^{\prime}<x ; \\
v h_{x, w^{\prime}}+h_{x^{\prime}, w^{\prime}}-\sum_{\substack{z \in \Lambda_{0}(w) \\
z \neq w}} \mu\left(z, w^{\prime}\right) h_{x, z}, & \text { if } x^{\prime}>x .
\end{array}\right.
$$

Finally, we note that (2.10) and (5.34) are the same equation. Summing up, we have categorified the recursive formula for Kazhdan-Lusztig polynomials given in (2.10).

\section{REFERENCES}

[BB06] Bjorner, A., \& Brenti, F. (2006). Combinatorics of Coxeter groups (Vol. 231). Springer Science \& Business Media.

[EW13] Elias, B., \& Williamson, G. (2013). Soergel calculus. arXiv preprint arXiv:1309.0865.

[EW14] Elias, B., \& Williamson, G. (2014). The Hodge theory of Soergel bimodules. Annals of Mathematics, 180 (3), 1089-1136.

[GL96] Graham, J. J., \& Lehrer, G. I. (1996). Cellular algebras. Inventiones mathematicae, 123(1), 1-34.

[HM10] Hu, J., \& Mathas, A. (2010). Graded cellular bases for the cyclotomic KhovanovLaudaRouquier algebras of type A. Advances in Mathematics, 225(2), 598-642.

[HU92] Humphreys, J. E. (1992). Reflection groups and Coxeter groups (Vol. 29). Cambridge university press.

[KL79] Kazhdan, D., \& Lusztig, G. (1979). Representations of Coxeter groups and Hecke algebras. Inventiones mathematicae, 53(2), 165-184.

[L08] Libedinsky, N. (2008). Sur la catgorie des bimodules de Soergel. Journal of Algebra, 320(7), 2675-2694.

[L15] Libedinsky, N. (2015). Light leaves and Lusztig's conjecture. Advances in Mathematics, 280, 772-807.

[P13] Plaza, D. (2013). Graded decomposition numbers for the blob algebra. Journal of Algebra, 394, 182-206.

[P14] Plaza, D. (2014). Graded cellularity and the Monotonicity Conjecture. arXiv preprint arXiv:1410.2136

[S92] Soergel, W. (1992). The combinatorics of Harish-Chandra bimodules. J. reine angew. Math, 429(49-74), 4.

[S97] Soergel, W. (1997). Kazhdan-Lusztig polynomials and a combinatoric for tilting modules. Representation Theory of the American Mathematical Society, 1(6), 83-114.

[S07] Soergel, W. (2007). Kazhdan-Lusztig polynomials and indecomposable bimodules over polynomial rings. J. Inst. Math. Jussieu, 6(3), 501-525.

[WI13] Williamson, G. (2013). Schubert calculus and torsion. arXiv preprint arXiv:1309.5055. 